\title{
A NUMERICAL STUDY OF RIEMANN PROBLEM SOLUTIONS AND STABILITY FOR A SYSTEM OF VISCOUS CONSERVATION LAWS OF MIXED TYPE*
}

\author{
MAHMOUD AFFOUF $\dagger$ AND RUSSEL E. CAFLISCH $\ddagger$
}

\begin{abstract}
A numerical study of the isothermal fluid equations with a nonmonotone equation of state (like that of van der Waals) and with viscosity and capillarity terms is presented. This system is ill-posed (i.e., elliptic in $x$ vs. $t$ ) in some regions of state space and well-posed (i.e., hyperbolic) in other regions. Thus, it may serve as a model for describing dynamic phase transitions. Numerical computations of phase jumps, shock waves, and rarefaction waves for this system are presented. Although the solution of the Riemann problem is not unique, all of these waves are found to be stable to infinitesimal initial perturbations. Criteria are found for instability after $O(1)$ initial perturbations. An analytic argument is made for stability of phase transitions.
\end{abstract}

Key words. Riemann problems, mixed type, conservation law, van der Waals' gas, phase transitions

AMS(MOS) subject classifications. 35L67, 76L05

1. Introduction. The isothermal fluid equations with viscosity and capillarity terms are

$$
\begin{gathered}
v_{t}-u_{x}=0 \\
u_{t}+p(v)_{x}=\varepsilon u_{x x}-\delta v_{x x x},
\end{gathered}
$$

in which $v$ is specific volume, $u$ is velocity, $x$ is the Lagrangian space variable, $\varepsilon$ is the viscosity coefficient, and $\delta$ is the capillarity coefficient. The capillarity term was first proposed by Korteweg [10] and later analyzed by Felderhof [2] and Bongiorno, Scriven, and Davis [1]. This system with a van der Waals pressure law was analyzed by Slemrod [19], [20] as a simple model for liquid-gas phase transitions.

The van der Waals pressure term is

$$
p(v)=\frac{R \theta}{v-b}-\frac{a}{v^{2}}
$$

for constants $a, b, R$, and $\theta$, which is sketched in Fig. 1(a). We will mainly use the simplified form

$$
p(v)=v-(v-2)^{3}
$$

sketched in Fig. 1(b). For these choices of $p$, there is an interval $[\alpha, \beta]$ of values of the specific volume $v$ for which $p^{\prime}(v)>0$. This unstable spinoidal region separates the two phases of liquid $(v<\alpha)$ and gas $(\beta<v)$. For $v \in[\alpha, \beta]$ the system (1.1) is elliptic in $x$ and $t$, while in the liquid or gas phases it is hyperbolic.

The system (1.1)-(1.3) is only a crude model for describing dynamic phase transition, without a solid physical basis. However, we believe that the mathematical 1990.

* Received by the editors December 27, 1988; accepted for publication (in revised form) February 15,

$\dagger$ Mathematics Department, New Jersey Institute of Technology, Newark, New Jersey 07102.

$\ddagger$ Mathematics Department, University of California, Los Angeles, Los Angeles, California 90024-1555. This research was supported in part by the Air Force Office of Scientific Research under University Research Initiative grant 86-0352 and grant 89-0003, and by National Science Foundation grant NSF-MCS-83-01260. 
(a)

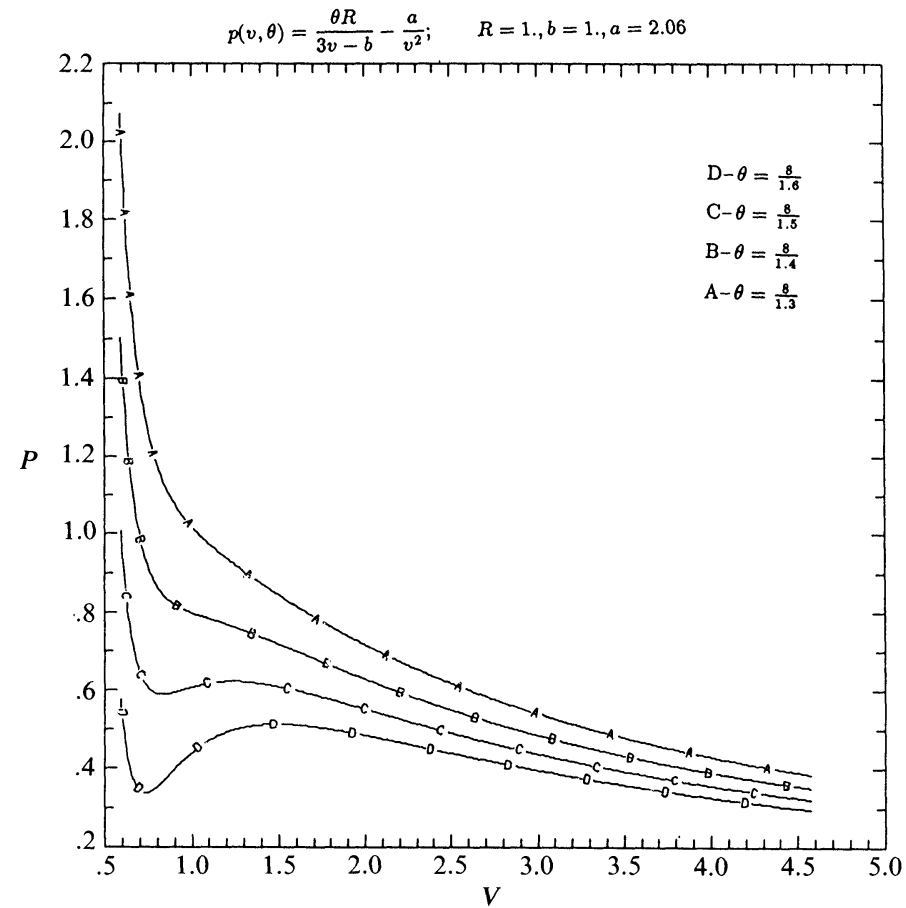

(b)

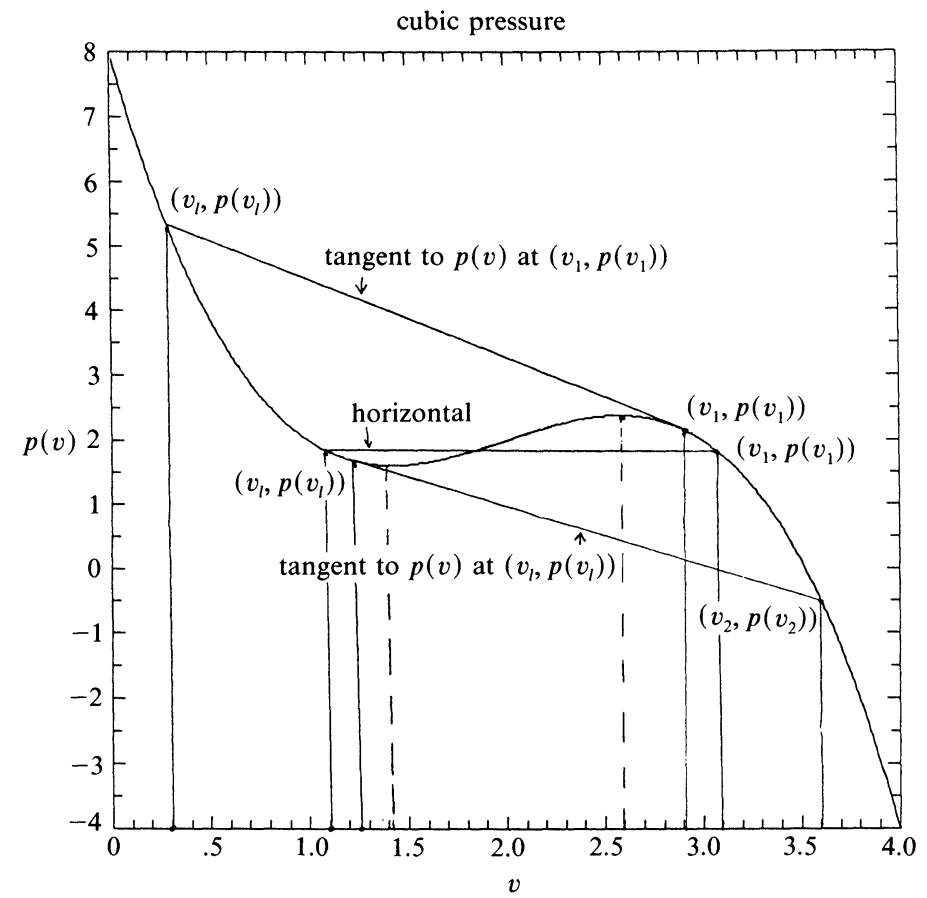

FIG. 1. (a) Van der Waals pressure for increasing values of temperature. (b) Cubic pressure law. 
properties of this system will occur in other more realistic phase transition models [3] or in other conservation laws of mixed type [14], [18].

Traveling wave solutions for (1.1)-(1.3) representing phase transitions and shock waves were analyzed by Slemrod [19], [20] and Hagan and Slemrod [5]. In particular, they showed that the capillarity term in (1.1) is necessary to get a full set of phase transition waves. Moreover, inclusion of capillarity eliminates some phase jumps and thus provides some uniqueness. These traveling wave solutions were then combined with rarefaction waves by Shearer [15]-[17] and Hattori [6], [7] to produce approximate solutions of the Riemann problem. The rarefaction waves would be exact solutions if $\varepsilon$ and $\delta$ were zero. By a Riemann problem solution, we mean any combination of traveling waves (shocks or phase transitions) and rarefactions that connect two given states $\left(u_{l}, v_{l}\right)$ at $x=-\infty$ and $\left(u_{r}, v_{r}\right)$ at $x=\infty$. A surprising result of Shearer [17] is that the Riemann problem solution for given end states $\left(u_{l}, v_{l}\right)$ and $\left(u_{r}, v_{r}\right)$ is not unique.

Viscosity and capillarity provide a selection principle for the nonunique weak solutions of the first-order part of (1.1). We use this because it seems more physically and mathematically correct than the alternatives, including chord conditions or hyperbolic numerical methods such as Lax-Friedrichs or Lax-Wendroff.

We present numerical computations of the initial value problem for $(1.1)$, with the pressure law (1.3) for rarefaction waves $(\S 5)$ and for shock waves and rarefaction waves ( $\$ \S 6$ and 7$)$. In particular, we show that all of these waves are stable to sufficiently small initial perturbations, if the end states of the wave are not in the spinoidal region. This may be surprising because of the nonuniqueness of Riemann problem solutions and because the phase transition waves pass smoothly through the spinoidal region. Stability of these phase transitions is explained by their narrow width $(\S 7.5)$. On the other hand, for moderate-sized initial perturbations, these waves may be unstable as described in $\$ \S 5$ and 7.

At present there is no mathematical analysis of stability of Riemann problem solutions for systems like (1.1) that are not strictly hyperbolic. Our numerical computations and resulting conclusions should be a useful guide for future analysis as well as further computations.

Related work on this system and other systems of mixed type has been performed by several authors: Nicolaenko [12] performed numerical solutions of (1.1) with periodic boundary conditions and analyzed inertial manifolds for this system. Slemrod and Flaherty [21] numerically solved (1.1) with $\varepsilon=\delta=0$ using the Lax-Friedrichs method, which provides numerical regularization terms. Slemrod and Marsden [22] analyzed chaos for (1.1) with forcing terms. General initial value problems have been discussed in [8], [23].

2. Basic properties of the system of mixed type. Some understanding of (1.1) is obtained by comparison with the equal area rule for equilibrium phase transitions and by analysis of the linearized equation for small perturbations of constant $u$ and $v$. First, note that in this Lagrangian formulation of the fluid equations, Galilean invariance means just that the equations are unchanged if a constant is added to the velocity $u$.

2.1. Equal area rule. An equilibrium phase transition has $u_{t}=u_{x}=v_{t}=0$. The equation for $v$ is then

$$
\delta v_{x x}+p(v)-p_{0}=0
$$

after integration once in $x$. A phase transition occurs if there is a (smooth) solution $v(x)$ of (2.1) with end states $v_{l}$ and $v_{r}$ in different phases. In particular, $p\left(v_{l}\right)=p\left(v_{r}\right)=p_{0}$. For $p_{1}<p_{0}<p_{2}$ there are stationary points $v_{1}, v_{2}$ for $(2.1)$ with $p\left(v_{1}\right)=p\left(v_{2}\right)=p_{0}$, but 
only for $p_{0}$ satisfying the equal area rule

$$
\int_{v_{1}}^{v_{2}}\left(p(v)-p_{0}\right) d v=0
$$

are $v_{1}$ and $v_{2}$ connected by a solution of (2.1). Condition (2.2) is easily derived by rewriting (2.1) as

$$
\delta w w_{v}+p(v)-p_{0}=0
$$

for $w=v_{x}$. Denote these "equal area" valves as $m=v_{1}$ and $M=v_{2}$. Also denote $\gamma$ and $\sigma$ satisfying $p(\gamma)=p(\alpha)$ and $p(\sigma)=p(\beta)$.

This derivation of the equal area rule (2.2) from the dynamic van der Waals fluid equations $(1.1)$ is independent of the choice of $p(v)$. It shows the importance of the capillarity term $\delta$ and demonstrates some consistency of (1.1) with equilibrium statistical mechanics.

A further insight into the system (1.1) for $\delta<\varepsilon^{2} / 4$ is gained by rewriting it as a viscous conservation system

$$
\begin{aligned}
& v_{t}-w_{x}=d_{1} v_{x x} \\
& w_{t}+p(v)_{x}=d_{2} w_{x x}
\end{aligned}
$$

in which

$$
\begin{aligned}
& w=u-d_{1} v_{x} \\
& d_{1}=\frac{1}{2}\left(\varepsilon-\sqrt{\varepsilon^{2}-4 \delta}\right) \\
& d_{2}=\frac{1}{2}\left(\varepsilon+\sqrt{\varepsilon^{2}-4 \delta}\right) .
\end{aligned}
$$

2.2. Dispersion relation for the linearized partial differential equation (PDE). The linearization of (1.1) around a constant state $v=v_{c}$ is

$$
v_{t t}+p^{\prime}\left(v_{c}\right) v_{x x}=\varepsilon v_{x x t}-\delta v_{x x x x} .
$$

This equation admits a solution in the form

$$
v(x, t)=\hat{v} e^{\lambda t+i k x},
$$

where $k$ is the wave number and $\lambda \in C$ is the frequency which is a complex value.

Substitute this function in (2.6) to obtain

$$
\lambda^{2}+\varepsilon k^{2} \lambda+\delta k^{4}-p^{\prime} k^{2}=0
$$

This equation is the dispersion relation for (2.6), which has two modes $\lambda_{+}$and $\lambda_{-}$, given by

$$
\lambda_{ \pm}=\frac{-\varepsilon k^{2} \pm \sqrt{\varepsilon^{2} k^{4}-4\left(\delta k^{4}-p^{\prime} k^{2}\right)}}{2} .
$$

The sign of the real part of $\lambda$ indicates the stability or the instability of the solution of (2.6). From (2.9) the solution is seen to be stable for $p^{\prime}<0$, because $\operatorname{Re}(\lambda)<0$. The only possible instability occurs for $\operatorname{Re}(\lambda)>0$, i.e., for $p^{\prime}>0$ with states in the elliptic region, and this can happen if

$$
\sqrt{\varepsilon^{2} k^{4}-4\left(\delta k^{4}-p^{\prime} k^{2}\right)}>\varepsilon k^{2}
$$

which can be simplified to

$$
|k|<\delta^{-1 / 2} \sqrt{p^{\prime}}
$$

This is the wave interval of instability. Figure 3 shows the real part of $\lambda$ versus the wave number $k$ for fixed $p^{\prime}>0, \varepsilon$, and $\delta$. 
The wave number $k_{m}$ with maximal rate of instability is found by differentiating $\lambda$ with respect to $k$ to obtain

$$
\frac{d \lambda}{d k}=-\varepsilon k+\frac{\beta k^{2}+2 p^{\prime}}{\sqrt{\beta k^{2}+4 p^{\prime}}},
$$

where $\beta=\varepsilon^{2}-4 \delta$. Then $d \lambda / d k=0$ for $k_{m}$ so that

$$
\varepsilon k_{m}=\frac{\beta k_{m}^{2}+2 p^{\prime}}{\sqrt{\beta k_{m}^{2}+4 p^{\prime}}} .
$$

(a)
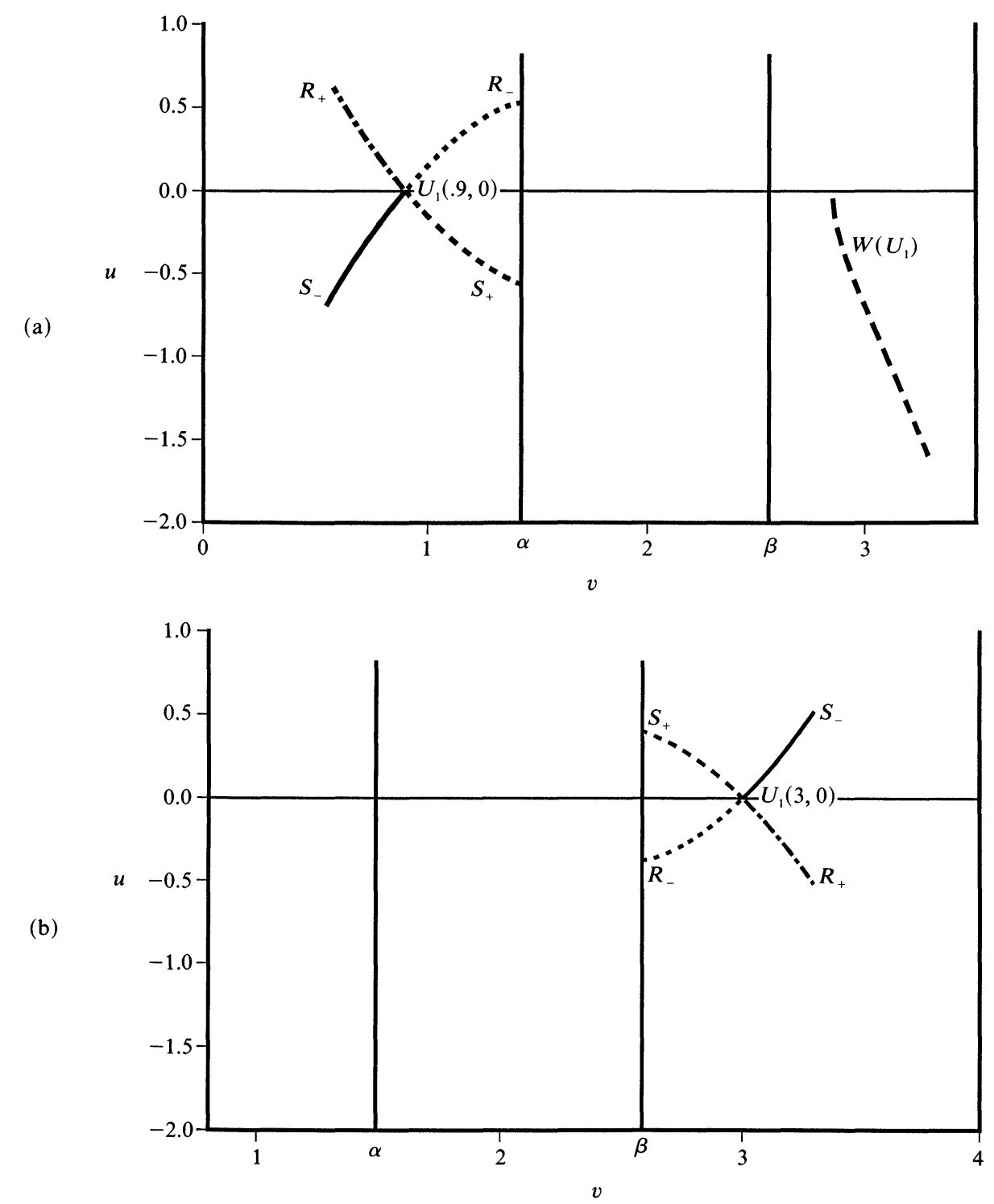

FIG. 2. Curve of points $U_{r}$ which are connected to a given point $U_{l}$ by a shock, phase jump, or rarefaction. (a) Shocks $S_{+}$and $S_{-}$, rarefactions $R_{+}$and $R_{-}$, and phase jumps $W\left(U_{l}\right)$ for $U_{l} \in H_{l}$. (b) Shock and rarefactions for $U_{l} \in H_{r}$. The phase jump curve $W\left(U_{l}\right)$ is omitted. 
There are three cases:

1. If $\beta=0$, then

$$
k_{m}=\sqrt{p^{\prime}} \varepsilon^{-1}
$$

2. If $\beta>0$, then

$$
k_{m}=\sqrt{(-2+\sqrt{4+\beta / \delta}) p^{\prime} / \beta} .
$$

For example, if $p^{\prime}=1$ and $\delta=c \varepsilon^{2}, c>\frac{1}{4}$, then

$$
k_{m}=\delta^{-1 / 2}\left(\frac{-2 c+\sqrt{c}}{1-4 c}\right)^{1 / 2} .
$$

3. If $\beta<0$, there is a similar formula for $k_{m}$.

2.3. Elementary waves. The system (1.1) has the following types of simple smooth solutions, which are similar to the elementary waves used for the Riemann problem:

1. The class of traveling wave (shock wave) solutions, denoted by $S_{+}^{\varepsilon, \delta}, S_{-}^{\varepsilon, \delta}$, which depend on $\varepsilon$ and $\delta$ and the shock speed $s$. If $\varepsilon$ is sufficiently large compared to $\delta$, these shock profiles are monotone; otherwise, they are oscillatory. The subscript indicates the direction of propagation of the shock. Omit the superscript $\delta$, if $\delta=0$.

2. The class of phase jumps, i.e., shock profiles connecting end states in different phases, denoted by $S_{+j}^{\varepsilon, \delta}$ and $S_{-j}^{\varepsilon, \delta}$.

3. The class of the stationary phase jumps, i.e., shock profiles with speed 0 , denoted by $S_{0}^{\varepsilon, \delta}$.

4. The class of the rarefaction waves depending on $\varepsilon, \delta$ denoted by $R_{+}^{\varepsilon, \delta}$ and $R_{-}^{\varepsilon, \delta}$.

For a given left end state $U_{\ell}=\left(u_{\ell}, v_{\ell}\right)$, the set of right end states $U_{r}=\left(u_{r}, v_{r}\right)$ to which $U_{\ell}$ can be connected by a single elementary wave is indicated in Fig. 2 . These elementary waves are described in detail in $\S \S 4$ and 5.

\section{The numerical method.}

3.1. The finite difference equation. Our goal is to investigate the behavior of the solution of the system (1.1) numerically. First, reduce the system (1.1) to an equivalent single equation. Since $u_{x t}=v_{t t}$ and $u_{x x x}=v_{t x x}$, the $t$ derivative of the second equation of $(1.1)$ is

$$
v_{t t}+p(v)_{x x}=\varepsilon v_{x x t}-\delta v_{x x x x} \quad t \geqq 0, x \in R^{1} .
$$

We will find solutions of (3.1) (or (1.1)) which are constant at $x= \pm \infty$. Equation (3.1) is preferable to the original system (1.1), since (1.1) requires a consistency condition for the jump $[u]=u_{+}-u_{-}$in terms of $[v]$. The system (1.1) could also be rewritten as an equation for $w$ with $w_{x}=v$ and $w_{t}=u$, but the resulting solutions $w$ would have linear growth at infinity.

Although we wish to solve the Cauchy problem for (3.1) on the whole real line, the numerical scheme must be solved on an interval $[a, b]$. Thus, in addition to initial conditions, we introduce artificial boundary conditions; i.e.,

$$
\begin{aligned}
& \left.v(x, t)\right|_{t=0}=v(x, 0)=f(x), \\
& \left.v_{t}(x, t)\right|_{t=0}=v_{t}(x, 0)=g(x), \\
& \left.v(x, t)\right|_{x=a}=\alpha_{a}(t), \\
& \left.v_{x}(x, t)\right|_{x=a}=\beta_{a}(t), \\
& \left.v(x, t)\right|_{x=b}=\alpha_{b}(t), \\
& \left.v_{x}(x, t)\right|_{x=b}=\beta_{b}(t) .
\end{aligned}
$$


Discretize the rectangular region $[a, b] \times[0, T]$ with space steps $\Delta x$ and time steps $\Delta t$, where $\Delta x=(b-a) / m$, and denote the approximate value of the solution $v(x, t)$ at the grid point with coordinate $(n \Delta t, j \Delta x)$ by $v_{j}^{n} \equiv v(n \Delta t, j \Delta x)$. Define the following finite difference operators:

$$
\begin{aligned}
& D_{x}^{+} v_{j}^{n}=v_{j+1}^{n}-v_{j}^{n}, \\
& D_{x}^{-} v_{j}^{n}=v_{j}^{n}-v_{j-1}^{n}, \\
& D_{x}^{0} v_{j}^{n}=v_{j+1}^{n}-v_{j-1}^{n}, \\
& D_{t}^{+} v_{j}^{n}=v_{j}^{n+1}-v_{j}^{n}, \\
& D_{t}^{-} v_{j}^{n}=v_{j}^{n}-v_{j}^{n-1}, \\
& D_{t}^{0} v_{j}^{n}=v_{j}^{n+1}-v_{j}^{n-1} .
\end{aligned}
$$

The following finite difference equation (FDE)

$$
\begin{aligned}
\frac{1}{\Delta t^{2}} D_{t}^{+} D_{t}^{-} v_{j}^{n} & +\frac{1}{\Delta x^{2}} D_{x}^{+} D_{x}^{-} p\left(v_{j}^{n}\right)-\frac{\varepsilon}{2 \Delta t \Delta x^{2}} D_{x}^{+} D_{x}^{-}\left(D_{t}^{0} v_{j}^{n}\right) \\
& +\frac{\delta}{2 \Delta x^{4}}\left(D_{x}^{+} D_{x}^{-}\right)^{2}\left(v_{j}^{n+1}+v_{j}^{n-1}\right)=0
\end{aligned}
$$

is consistent with the PDE (3.1), with global discretization error

$$
E(\Delta x, \Delta t)=O\left(\Delta t^{2}\right)+O\left(\Delta x^{2}\right) .
$$

3.2. Linearized stability of the numerical scheme. To analyze the linear stability of the numerical method, denote the error as

$$
E_{j}^{n}=v(n \Delta t, j \Delta x)-v_{j}^{n},
$$

in which $v(n \Delta t, j \Delta x)$ is the exact value of the solution of (3.1) at the point with coordinate $(n \Delta t, j \Delta x)$ and $v_{j}^{n}$ is the solution of the numerical scheme (3.4). This approximately solves the following linearized equation (with boundary conditions omitted):

$$
\begin{aligned}
& \frac{1}{\Delta t^{2}} D_{t}^{+} D_{t}^{-} E_{j}^{n}+\frac{p^{\prime}}{\Delta x^{2}} D_{x}^{+} D_{x}^{-} E_{j}^{n} \\
& \quad-\frac{\varepsilon}{2 \Delta t \Delta x^{2}} D_{x}^{+} D_{x}^{-} D_{t}^{0} E_{j}^{n}+\frac{\delta}{2 \Delta x^{4}}\left(D_{x}^{+} D_{x}^{-}\right)^{2}\left(E_{j}^{n+1}+E_{j}^{n-1}\right)=0 .
\end{aligned}
$$

Suppose

$$
E_{j}^{n}=\xi^{n} e^{i j \beta \Delta x}
$$

where $\xi=e^{\alpha \Delta t}$, and $\alpha$ is analogous to the frequency and $\beta$ to the wave number. Then $\xi$ satisfies the following quadratic equation.

$$
\begin{aligned}
(1+ & \left.2 \varepsilon r \sin ^{2} \frac{\beta \Delta x}{2}+8 \delta r^{2} \sin ^{4} \frac{\beta \Delta x}{2}\right) \xi^{2} \\
& +\left(-2-4 p^{\prime} h^{2} \sin ^{2} \frac{\beta \Delta x}{2}\right) \xi \\
& +\left(1-2 \varepsilon r \sin ^{2} \frac{\beta \Delta x}{2}+8 \delta r^{2} \sin ^{4} \frac{\beta \Delta x}{2}\right)=0
\end{aligned}
$$


in which $h=\Delta t / \Delta x$ and $r=\Delta t / \Delta x^{2}$. The numerical scheme (3.9) is stable if $|\xi| \leqq 1$. For $p^{\prime}<0$ a straightforward analysis shows this to be the case for all $\varepsilon \geqq 0, \delta \geqq 0$, $\Delta x>0$, and $\Delta t>0$ with

$$
(\Delta t / \Delta x)^{2}<\frac{1}{-p^{\prime}}
$$

which is the Courant-Friedrichs-Lewy (CFL) condition.

For $p^{\prime}>0$, i.e., for states in the unstable elliptic region, the dispersion relation for the FDE is a good approximation to that of the PDE in the interval of instability (2.10). This agreement is illustrated in Fig. 3, where the real part of $\lambda$ of the linearized PDE (2.6) and the frequency $\alpha=\ln (|\xi|) / \Delta t$ of the FDE (3.9) are plotted versus the wave number $k$, for fixed values of $p^{\prime}>0, \varepsilon, \delta, \Delta x$, and $\Delta t$ satisfying the CFL condition (3.10). The agreement between these dispersion relations indicates that the FDE should correctly simulate the instability of the PDE, and gives us confidence that the solution of the FDE (3.4) will converge to the exact solution of the PDE (3.1).

3.3. Numerical boundary conditions. This proposed scheme is implicit, multilevel, and of order $O\left(\Delta t^{2}+\Delta x^{2}\right)$. Therefore, it is essential to define the solution at an additional point outside the boundary, in addition to using the given boundary conditions. At $x=a-\Delta x$, extrapolate to get the value of $v$ as a linear combination

$$
v(a-\Delta x)=\alpha v(x)+\beta v(x+\Delta x)+\gamma v(x+2 \Delta x)+\sigma \Delta x v^{\prime}(x) \text {. }
$$

This approximation is valid up to $O\left(\Delta x^{4}\right)$ if

$$
\alpha=-\frac{3}{2}, \quad \beta=3, \quad \gamma=-\frac{1}{2}, \quad \sigma=-3,
$$

so that at the left end we use

$$
v(a-\Delta x)=-\frac{3}{2} v(a)+3 v(a+\Delta x)-\frac{1}{2} v(a+2 \Delta x)-3 v^{\prime}(a) \Delta x+O\left(\Delta x^{4}\right)
$$

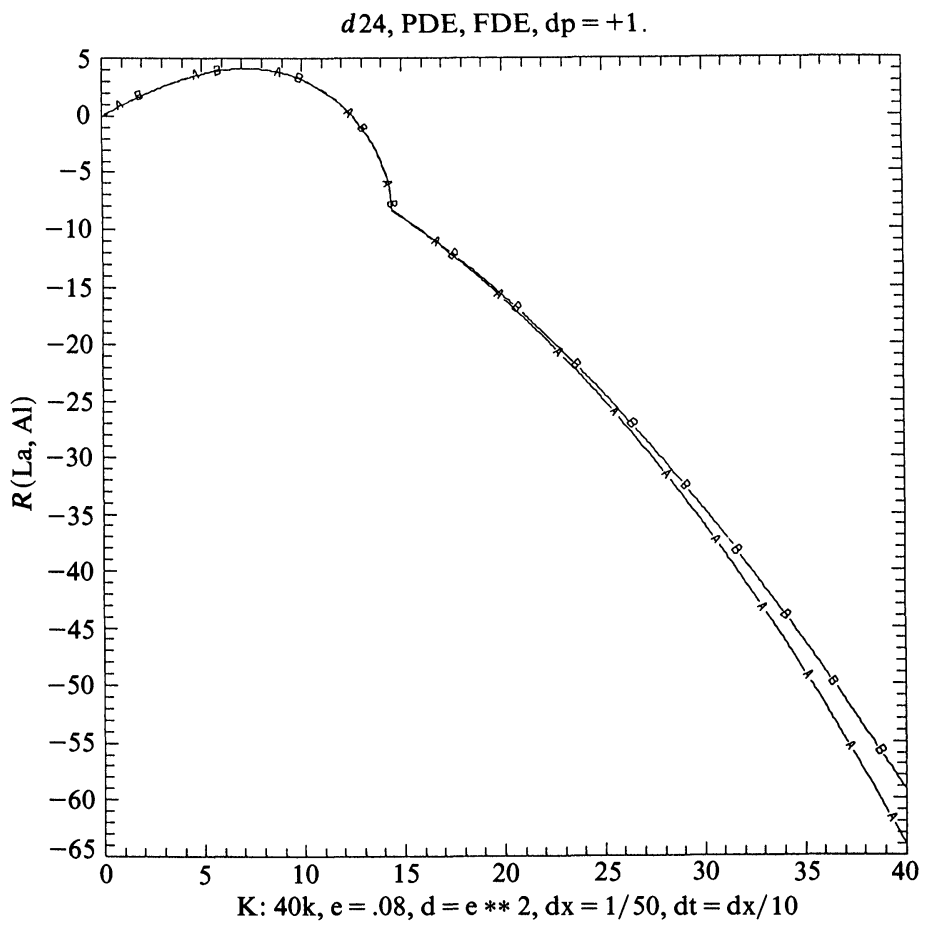

FIG. 3. Real part of $\lambda$ (growth rate) vs. wave number $k$ for the PDE and the FDE. 
and similarly at the right end $b=(m+1) \Delta x$ we use

$$
v(b+\Delta x)=-\frac{3}{2} v(b)+3 v(b-\Delta x)-\frac{1}{2} v(b-2 \Delta x)+3 v^{\prime}(b) \Delta x+O\left(\Delta x^{4}\right) .
$$

3.4. Initialization. Since the numerical scheme (3.4) involves two previous time levels, an approximation for $v(\Delta t, \cdot)$ is needed. Expand $v(\Delta t, \cdot)$ in a Taylor series as

$$
v(\Delta t, \cdot)=v(0, \cdot)+v^{\prime}(0, \cdot) \Delta t+v^{\prime \prime}(0, \cdot) \frac{\Delta t^{2}}{2}+O\left(\Delta t^{3}\right)
$$

The first two terms $v(0, \cdot)$ and $v^{\prime}(0, \cdot)$ are given as initial data, while the value $v^{\prime \prime}(0, \cdot)$ is found from (3.1) to be

$$
v_{t t}(0, \cdot)=-p(v(0, \cdot))_{x x}+\varepsilon v(0, \cdot)_{x x t}-\delta v(0, \cdot)_{x x x x} .
$$

3.5. Choice of initial data. We would like to solve (1.1) with the following Riemann data

$$
(u, v)(x, 0)= \begin{cases}\left(u_{l}, v_{l}\right), & x<0 \\ \left(u_{r}, v_{r}\right), & x>0 .\end{cases}
$$

Since $s^{2}=-[p] /[v]$ and $v_{t}=u_{x}$, then $v_{t}(x, 0)$ is a $\delta$-function, which is approximated as

$$
v_{t}(x, 0)=u_{x}(x, 0)= \begin{cases}0, & x<-x_{0} \\ h / 2, & x=-x_{0} \\ h, & -x_{0}<x<x_{0} \\ h / 2, & x=x_{0} \\ 0, & x_{0}<x\end{cases}
$$

where the height $h$ is defined by

$$
\int_{-\infty}^{+\infty} u_{x}(x, 0)=-s\left(v_{r}-v_{l}\right)=h \cdot 2 x_{0},
$$

with $s^{2}=-[p] /[v]$. We use $x_{0}=\Delta x$.

The viscosity and capillarity in (3.1) are found to rapidly smooth the solution so that the smallest important wavelength is at least of size $\sqrt{\delta}$. So we simplify the computation by using the following smoothed initial data:

$$
v(x, 0)=\frac{v_{l}+v_{r}}{2}-\frac{v_{r}-v_{l}}{2} \tanh \left(\frac{x}{\sqrt{\delta}}\right) .
$$

3.6. Numerical parameters. The computations described below have been performed for a range of values of $\varepsilon$ and $\delta$. The results in all of the following figures are for $\varepsilon=.02$ and $\delta=\varepsilon^{2}$, except when indicated otherwise. The computation is found to converge if $\Delta x$ is much smaller than the shock width and if $\Delta t$ satisfies the CFL condition. For these values of $\varepsilon$ and $\delta$, we use $\Delta x=.01$ and $\Delta t=.001$.

The significant values of $v$ (defined in $\S \S 1$ and 2.1 ) are

$$
\begin{gathered}
\alpha=1.422, \quad \beta=2.577, \quad \gamma=.845, \\
m=1, \quad M=3 .
\end{gathered}
$$

4. Rarefaction waves. The rarefaction waves make sense only in the hyperbolic regions $H_{l}$ and $H_{r}$. Thus, suppose the initial data $U_{l}=\left(u_{l}, v_{l}\right)$ and $U_{r}=\left(u_{r}, v_{r}\right)$ are in $H_{r}$ and are connected by rarefactions $R_{-}^{\varepsilon, \delta}, R_{+}^{\varepsilon, \delta}$, or $R_{-}^{\varepsilon, \delta} R_{+}^{\varepsilon, \delta}$. 


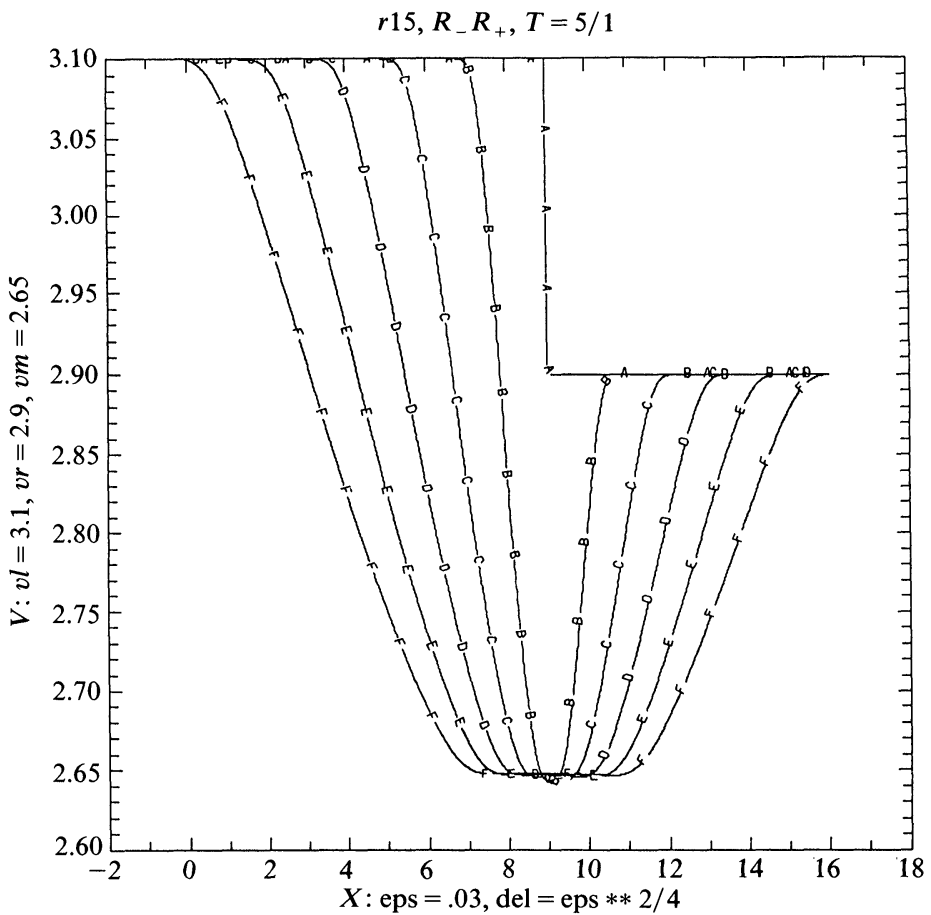

FIG. 4. A solution with two rarefaction waves. In this and subsequent figures, the curves labeled A, B, C, $\mathrm{D}, \mathrm{E}$ are the solution at successive (increasing) times that are indicated $(5 / 1$ means $1,2,3,4,5)$.

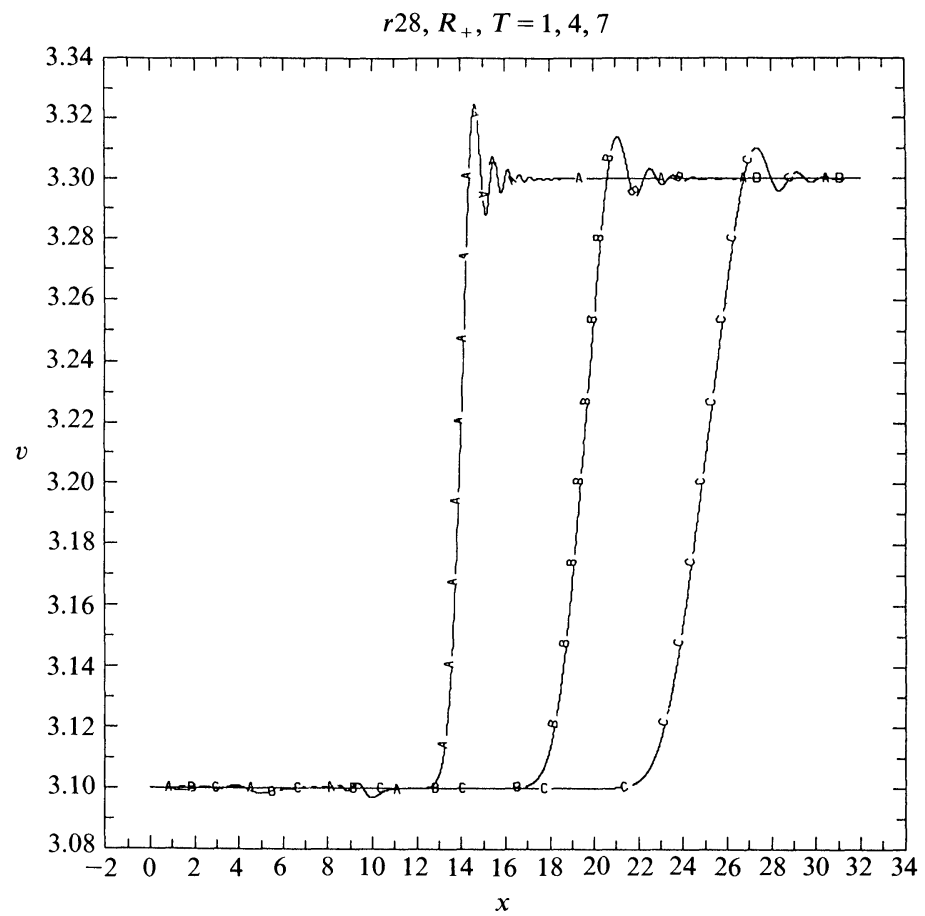

FIG. 5. A rarefaction wave with a decaying diffusion wave on the left and a dispersing oscillation on the right. 
Computations with such initial data recover these time dependent solutions. Figure 4 shows a solution with two rarefactions. In Fig. 5 the solution $R_{+}^{\varepsilon, \delta}$ is plotted showing a forward rarefaction with a small decaying diffusion wave on the left and a dispersing oscillation on the right. In both cases, the diffusion term dominates the dispersion term. This computation has been performed at a sequence of values of $\Delta x$ and $\Delta t$ that are small enough to guarantee that the oscillations are not a numerical artifact and are well resolved.

4.1. Stability of rarefaction waves. If the end states $v_{l}, v_{r}$ are far from the elliptic region $[\alpha, \beta]$, then the rarefaction waves connecting these states are observed to be stable with respect to any small perturbations. However, if one state of the initial data (4.4) is very close to the elliptic region, then for any small perturbation entering the elliptic region, the connection between the end states is unstable. This is because at the end point which is close to the elliptic region, the speed of the flow equals $p^{\prime}$, which is nearly zero, so that the motion is almost stationary. Thus, any perturbation entering the elliptic region creeps to the other phase, and, as a result, a different connection is obtained. For example, if the solution is initially a backward rarefaction $\boldsymbol{R}_{-}^{\varepsilon, \delta}$ with $v_{r}$ very close to $\beta$, then in finite time the solution becomes $\boldsymbol{R}_{-}^{\varepsilon, \delta} \boldsymbol{S}_{-}^{\varepsilon, \delta} \boldsymbol{S}_{-j}^{\varepsilon, \delta} \boldsymbol{S}_{+j}^{\varepsilon, \delta} \boldsymbol{S}_{+}^{\varepsilon, \delta}$. This instability also occurs when there is no dispersion $(\delta=0)$, but then the final result is $\boldsymbol{R}_{-}^{\varepsilon} \boldsymbol{S}_{0}^{\varepsilon} \boldsymbol{S}_{0}^{\varepsilon}$.

Suppose that the connection between $U_{l}, U_{r}$ consists of two rarefactions, i.e., there is a state $U_{m}$ such that the connection $U_{l}, U_{m}$ is a backward rarefaction and the connection $U_{m}, U_{r}$ is a forward rarefaction. Take initial data as in $\S 3.5$ and take $U_{m}$ very close to the elliptic region. Then the solution is unstable, and the two rarefactions become $R_{-}^{\varepsilon, \delta} S_{-}^{\varepsilon, \delta} S_{-j}^{\varepsilon, \delta} S_{+j}^{\varepsilon, \delta} S_{+}^{\varepsilon, \delta} R_{+}^{\varepsilon, \delta}$, as shown in Fig. 6. Also, if $\delta=0$ then the solution is still unstable, but the final connection is $R_{-}^{\varepsilon} S_{0}^{\varepsilon} S_{0}^{\varepsilon} R_{+}^{\varepsilon}$.

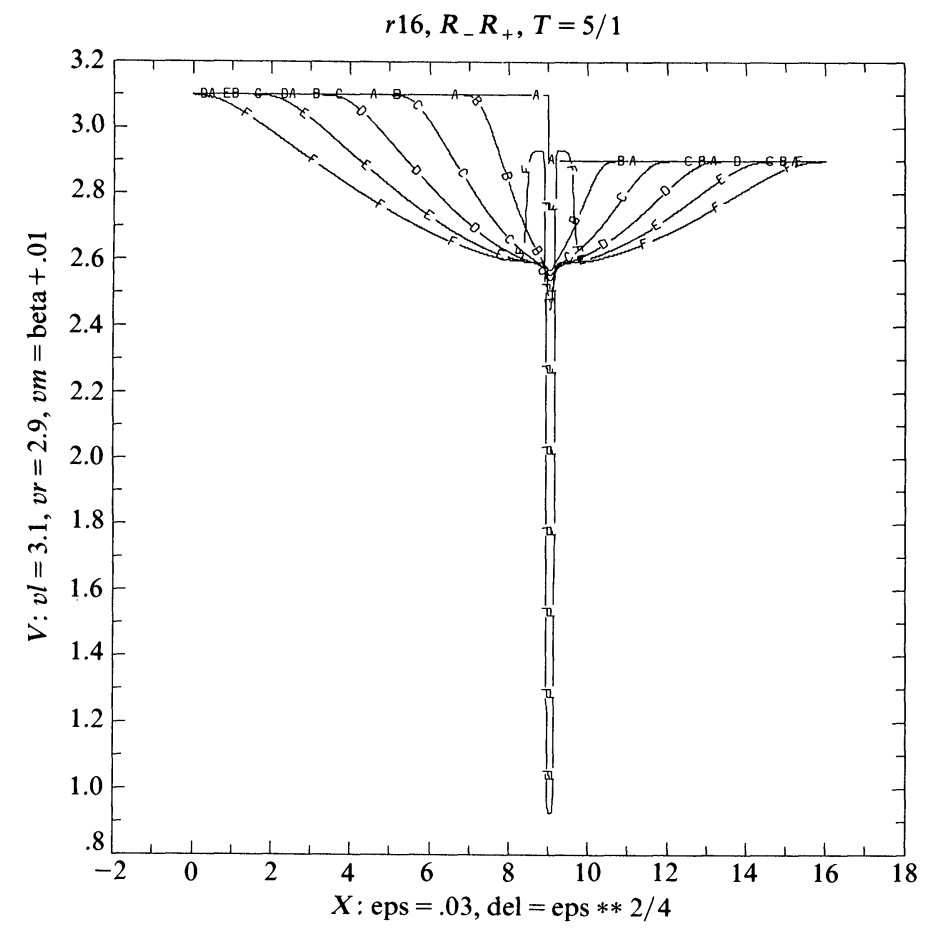

FIG. 6. Instability of two rarefactions with a state close to the elliptic region. 
Two states within the same phase $H_{r}$ may also be connected by a rarefaction followed by a phase jump to $H_{l}$ and a second phase jump back to $H_{r}$. Such a solution of the form $R_{-}^{\varepsilon, \delta} S_{-j}^{\varepsilon, \delta} S_{+j}^{\varepsilon, \delta} R_{+}^{\varepsilon, \delta}$, which crosses the elliptic region twice, is shown in Fig. 7. For $\delta=0$ a similar solution of the form $R_{-}^{\varepsilon} S_{-j}^{\varepsilon} S_{+j}^{\varepsilon} R_{+}^{\varepsilon}$, is presented in Fig. 8 . In the latter case, the speed of the phase jump is equal to the speed of the adjacent rarefaction wave.

5. Traveling wave solutions: Shocks and phase jumps. In this section exact traveling wave solutions for the equation (1.1) are described and classified, based on a numerical investigation of their properties. First, a distinction is made between traveling waves that are shocks and those that are phase jumps.

5.1. Shock waves. Assume that the end states $U_{l}$ and $U_{r}$ are in the same phase, either $H_{l}$ or $H_{r}$, and that the connection between $U_{l}$ and $U_{r}$ is either a backward shock, a forward shock, or a combination of them. The solution of (4.1) with initial data (4.4) will be approximated by the corresponding shock profiles $S_{-}^{\varepsilon, \delta}, S_{+}^{\varepsilon, \delta}$, or $S_{-}^{\varepsilon, \delta} S_{+}^{\varepsilon, \delta}$. If viscosity dominates dispersion, then the shock profiles are monotone functions; while if dispersion dominates viscosity, then the solutions have oscillations around the corresponding spiral node. These traveling wave solutions solve an autonomous ordinary differential equation and will be oscillatory when one of the endpoints is a spiral node, which is the case if

$$
\delta>s^{2}\left(p^{\prime}(v)+s^{2}\right)^{-1} \varepsilon^{2} / 4
$$

as discussed by Whitham [26, p. 482]. In case of a combination of two shocks, the oscillations are in front of each profile, whether forward or backward. Figure 9 shows a combination of two monotone shock profiles, and Fig. 10 shows a combination of two oscillatory shock profiles.

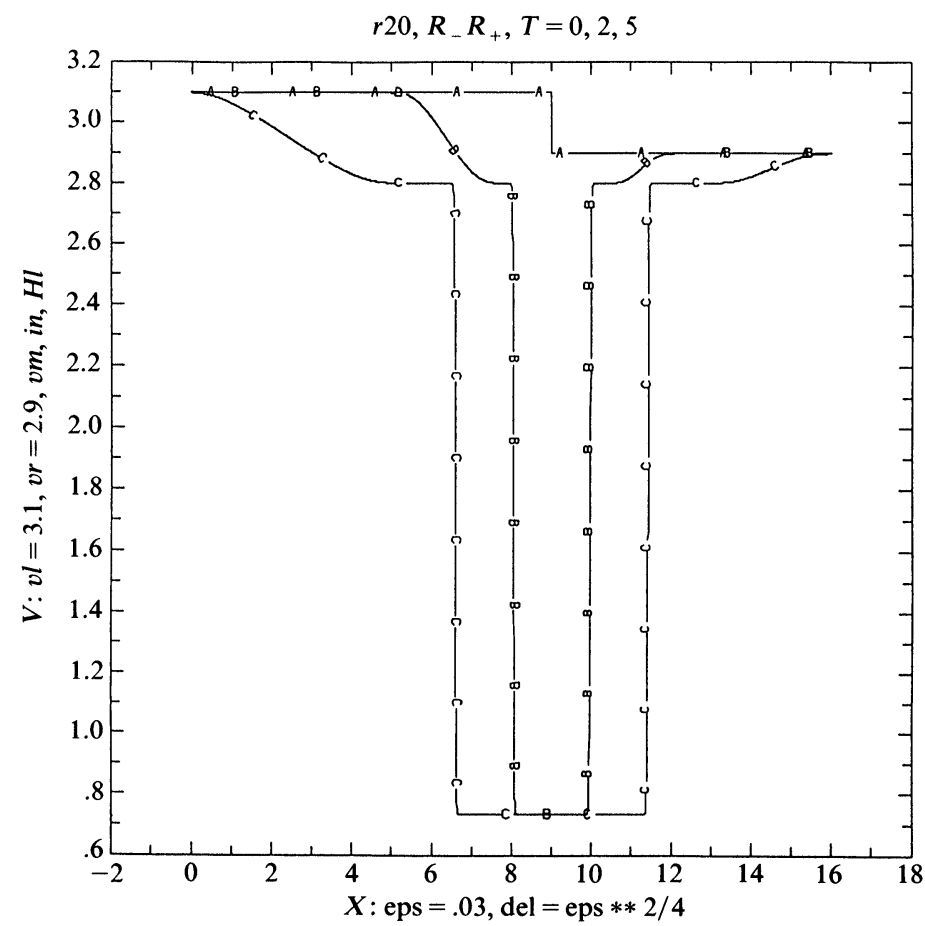

FIG. 7. Two states $U_{l}$ and $U_{r}$ in $H_{r}$ connected by a rarefaction followed by two phase jumps, for $\delta \neq 0$. 


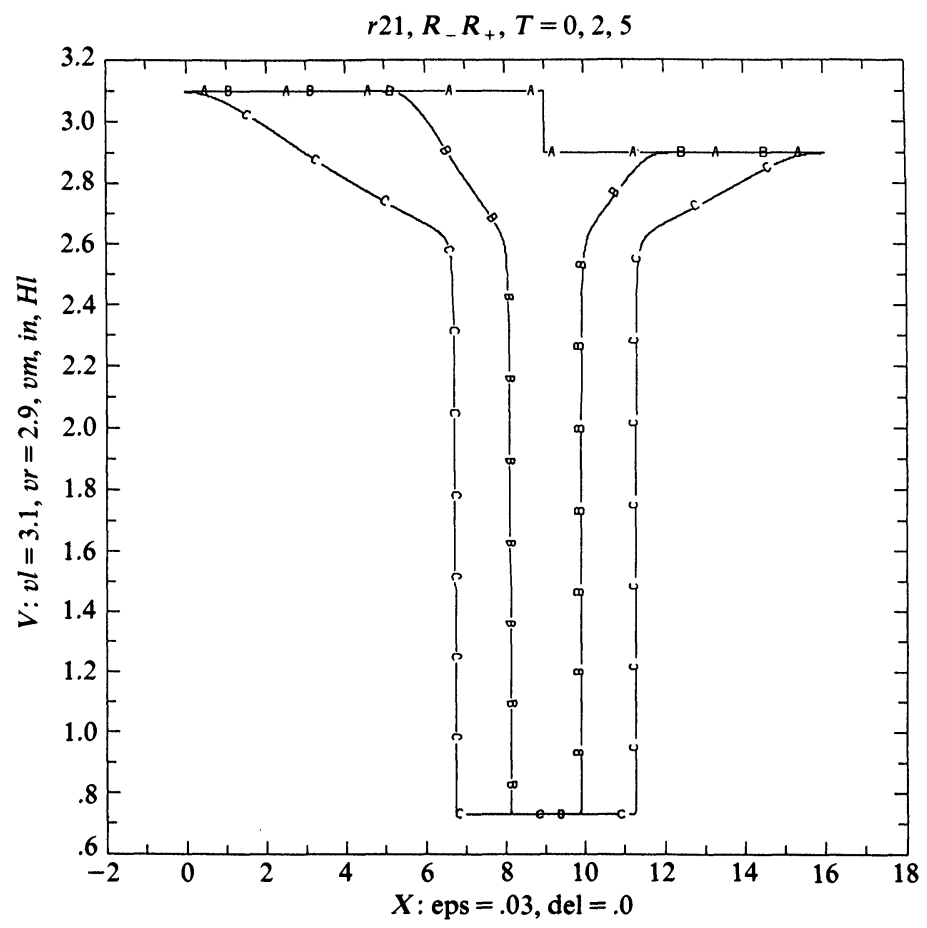

FIG. 8. Same as Fig. 7, but with $\delta=0$.

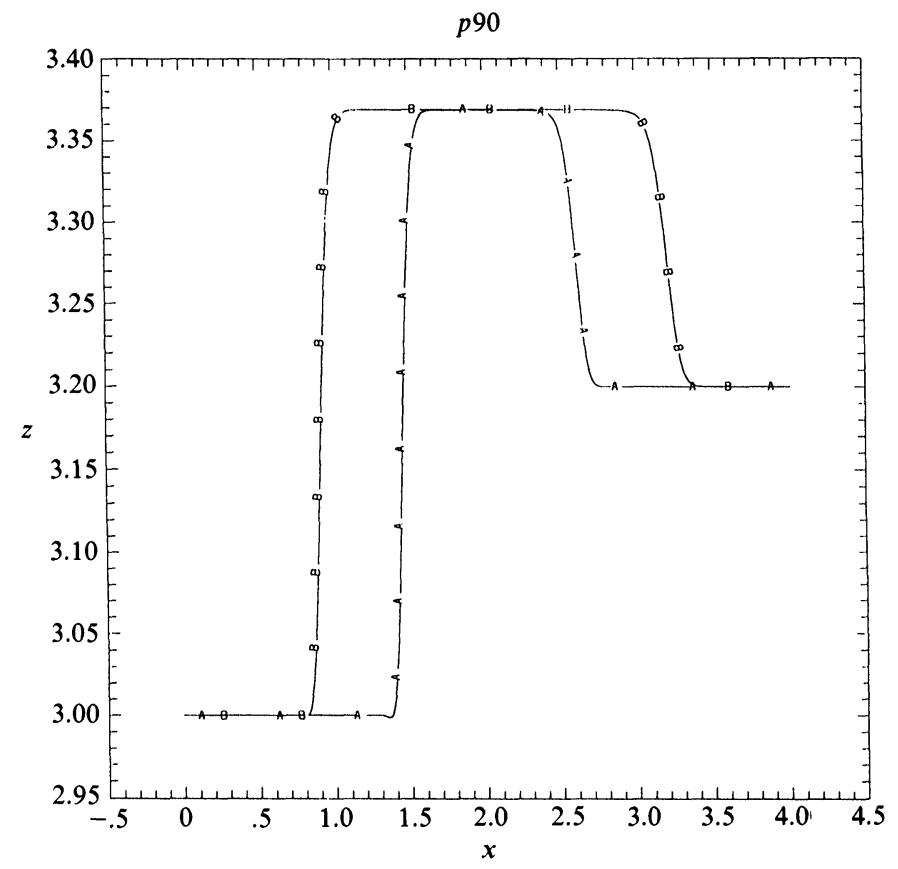

FIG. 9. A combination of two monotone shocks. 


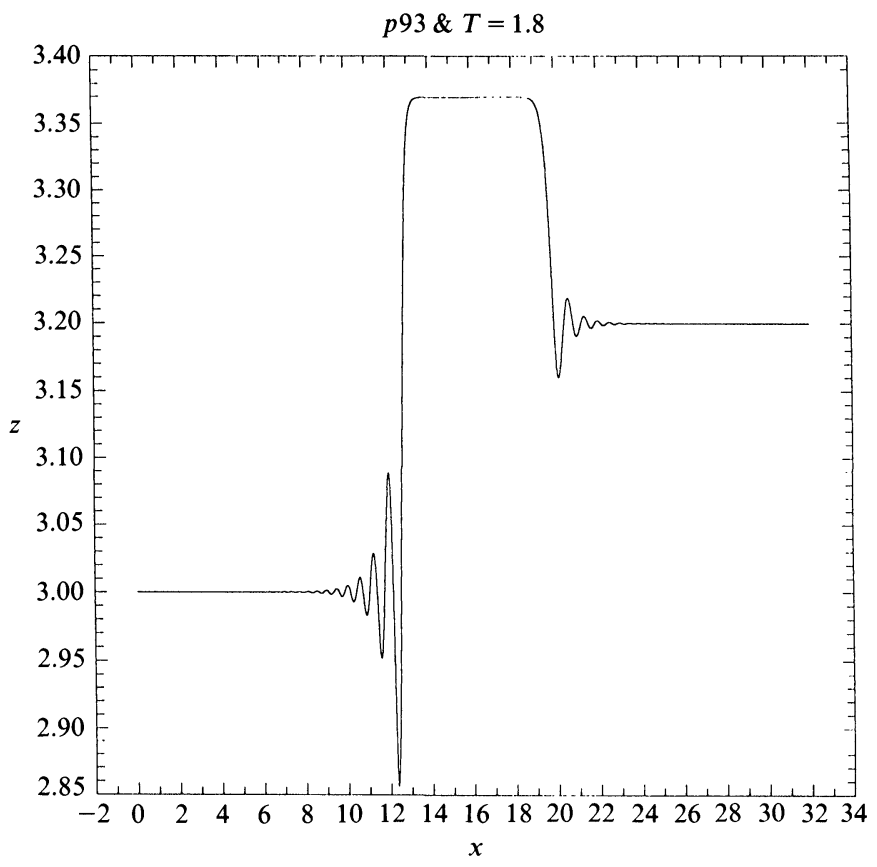

FIG. 10. A combination of two oscillatory shocks.

5.2. Phase jumps. We next consider Riemann data with end states in different phases, i.e., $U_{l} \in H_{l}$ and $U_{r} \in H_{r}$. First, make some definitions and notations.

1. We call the solution of (4.1) with initial data (4.4) a connection, and denote it by $\left\langle U_{l} \rightarrow U_{r}\right\rangle$.

2. We divide the liquid phase, i.e., $H_{l}$ (in which the end state $U_{l}$ is located), into three distinct regions:

$$
D_{1}=\{(u, v): v<\gamma\},
$$

which is the globally attractive region,

$$
D_{2}=\{(u, v): \gamma \leqq v \leqq m\},
$$

which is the dispersively stable region, and

$$
D_{3}=\{(u, v): m<v<\alpha\},
$$

which is the metastable region.

3. The stationary point $U_{s t}=\left(u_{s t}, v_{s t}\right) \in H_{r}$ is defined for $U_{l} \in D_{2}$ to satisfy

$$
u_{s t}=u_{l}, \quad p\left(v_{s t}\right)=p\left(v_{l}\right) \quad \text { and } \quad p^{\prime}\left(v_{s t}\right)<0 .
$$

There is a stationary solution connecting $U_{l}$ and $U_{s t}$ if $\delta=0$, but if $\delta \neq 0$ the only such connection is that of the equal area rule (cf. 6 below).

4. The tangential point $U_{t}=\left(u_{t}, v_{t}\right)$ is defined for $U_{l} \in D_{1}$ as follows: the line passing through $\left(p\left(v_{l}\right), v_{l}\right)$ and $\left(p\left(v_{t}\right), v_{t}\right)$ is tangential to the curve $p(v)$ at the point $\left(p\left(v_{t}\right), v_{t}\right)$, and the jump $U_{l}$ to $U_{t}$ is permissible.

5. The admissible point $U^{*}=\left(u^{*}, v^{*}\right)$ is defined for $U_{l}$ in some subset of $D_{1} \cup D_{2}$ including $v_{l}=m$, according to the viscosity-capillarity criteria so that $\left\langle U_{l} \rightarrow U^{*}\right\rangle$ is the unique connection that is a forward phase jump, denoted by $S_{j}^{\varepsilon, \delta}$. 
6. The equal area point $U_{e q}=\left(u_{e q}, v_{e q}\right)$ is defined to satisfy

$$
\begin{gathered}
-s\left(v_{e q}-v_{l}\right)=\left(u_{e q}-u_{l}\right) \\
\int_{v_{l}}^{v_{e q}}\left(p(v)-p\left(v_{l}\right)+s^{2}\left(v-v_{l}\right)\right) d v=0 .
\end{gathered}
$$

7. The geometric locus $W\left(U_{l}\right)$ is the Rankine-Hugoniot curve in the phase $H_{r}$ with respect to $U_{l}$, defined as follows:

$$
W\left(U_{l}\right)=\left\{(u, v):-s\left(v-v_{l}\right)=\left(u-u_{l}\right) \text { and } s^{2}=-\frac{[p]}{[v]} ; s>0\right\} .
$$

The endpoints of $W\left(U_{l}\right)$ are $U_{e q}$ and either $U_{s t}$ for $U_{l} \in D_{2}$ or $U_{t}$ for $U_{l} \in D_{1}$. This is plotted in Fig. 2.

In addition to these definitions, we will assume that $U_{l}=\left(0, v_{l}\right) \in H_{l}$ and $U_{r}=$ $\left(u_{r}, v_{r}\right) \in H_{r}$. From an extensive series of computations we have been able to classify the possible connections $\left\langle U_{l} \rightarrow U_{r}\right\rangle$ for fixed $\varepsilon, \delta$.

5.3. Classification. First, we restrict ourselves to forward phase jumps. We have identified the following 12 types of solutions of the system (4.1) with Riemann initial data as in $\S 3.5$ :

$\begin{aligned} \text { 1. } & S_{0}^{\varepsilon, \delta}, S_{0}^{\varepsilon}, & \text { 2. } & S_{j}^{\varepsilon, \delta}, \\ \text { 3. } & S_{-}^{\varepsilon, \delta} S_{j}^{\varepsilon, \delta} S_{+}^{\varepsilon, \delta}, & \text { 4. } & S_{-}^{\varepsilon, \delta} S_{j}^{\varepsilon, \delta} R_{+}^{\varepsilon, \delta}, \\ \text { 5. } & R_{-}^{\varepsilon, \delta} S_{j}^{\varepsilon, \delta} S_{+}^{\varepsilon, \delta}, & \text { 6. } & R_{-}^{\varepsilon, \delta} S_{j}^{\varepsilon, \delta} R_{+}^{\varepsilon, \delta}, \\ \text { 7. } & S_{-}^{\varepsilon} S_{0}^{\varepsilon} R_{+}^{\varepsilon}, & \text { 8. } & S_{-}^{\varepsilon} S_{j}^{\varepsilon} R_{+}^{\varepsilon}, \\ \text { 9. } & S_{-}^{\varepsilon, \delta} S_{j}^{\varepsilon, \delta}, & 10 . & R_{-}^{\varepsilon, \delta} S_{j}^{\varepsilon, \delta} \\ 11 . & S_{j}^{\varepsilon, \delta} S_{+}^{\varepsilon, \delta}, & 12 . & S_{j}^{\varepsilon, \delta} R_{+}^{\varepsilon, \delta} .\end{aligned}$

Several of these possibilities are plotted in Figs. 11-14.

Based on our computations we believe that these 12 are all the possibilities, as argued next. In particular, for $\delta \neq 0$ and for two end states in different phases, we argue that there can be at most one phase jump; while for two states in the same phase there can be either two or no phase jumps. The validity of this classification is based partly on analytic information and partly on numerical results. These are summarized as follows:

(1) The solution connecting $U_{l}$ to $U_{r}$ may cross the elliptic region only as a phase jump, i.e., $S_{j}^{\varepsilon, \delta}$ or $S_{0}^{\varepsilon, \delta}$, since there are no rarefaction waves that cross the elliptic region.

(2) For two states that are connected by a forward phase jump, there are only several possibilities. The left state, which is in $H_{l}$, can be connected to another state in the same phase by $S_{-}^{\varepsilon, \delta}$ or $R_{-}^{\varepsilon, \delta}$, and the right state in $H_{r}$ can be connected to another state in the same phase by $S_{+}^{\varepsilon, \delta}$ or $R_{+}^{\varepsilon, \delta}$. This is because these connections are in the same hyperbolic region and the speed of the phase jump is smaller than any forward shock or rarefaction wave that may connect such end states.

(3) According to the viscosity-capillarity admissibility criteria, for any fixed $\varepsilon, \delta$ and $U_{l} \in D_{1} \cup D_{2}$ there exists a unique $U^{*}$ such that $\left\langle U_{l} \rightarrow U^{*}\right\rangle=S_{j}^{\varepsilon, \delta}$. This unique point is located on the geometric locus $W\left(U_{l}\right)$ as shown by Slemrod [20].

Furthermore, our numerical results show monotone behavior of the function $U^{*}=\left(u^{*}, v^{*}\right)$, depending on $\varepsilon, \delta$ and $U_{l}=\left(u_{l}, v_{l}\right)$, as described next.

(3a) If $U_{l} \in D_{2}$, then $U^{*}$ is an increasing function of $U_{l}$; i.e., each component of $U^{*}$ is an increasing function of the corresponding component of $U_{l}$. Also, for fixed $U_{l}, U^{*}$ varies on $W\left(U_{l}\right)$ as $\varepsilon$ and $\delta$ vary, with $v^{*}$ a decreasing function of $\varepsilon$ for fixed 


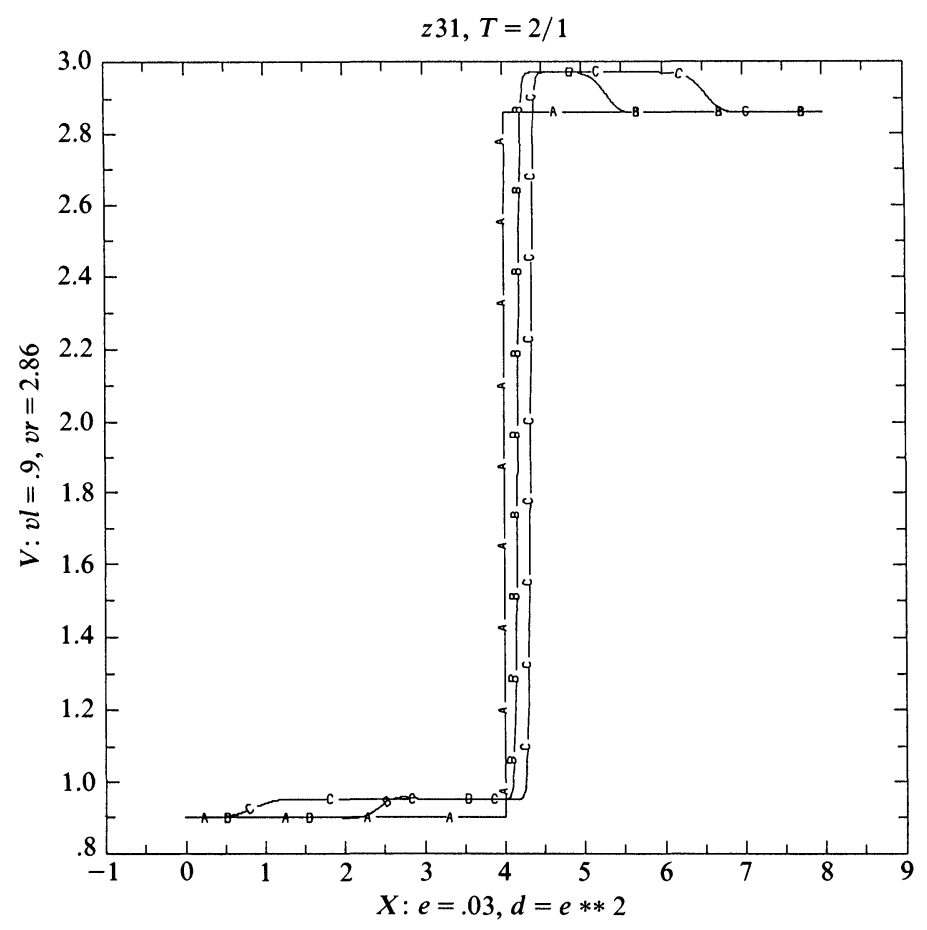

FIG. 11. A connection $R_{-}^{\varepsilon, \delta} S_{+j}^{\varepsilon, \delta} S_{+}^{\varepsilon, \delta}$.

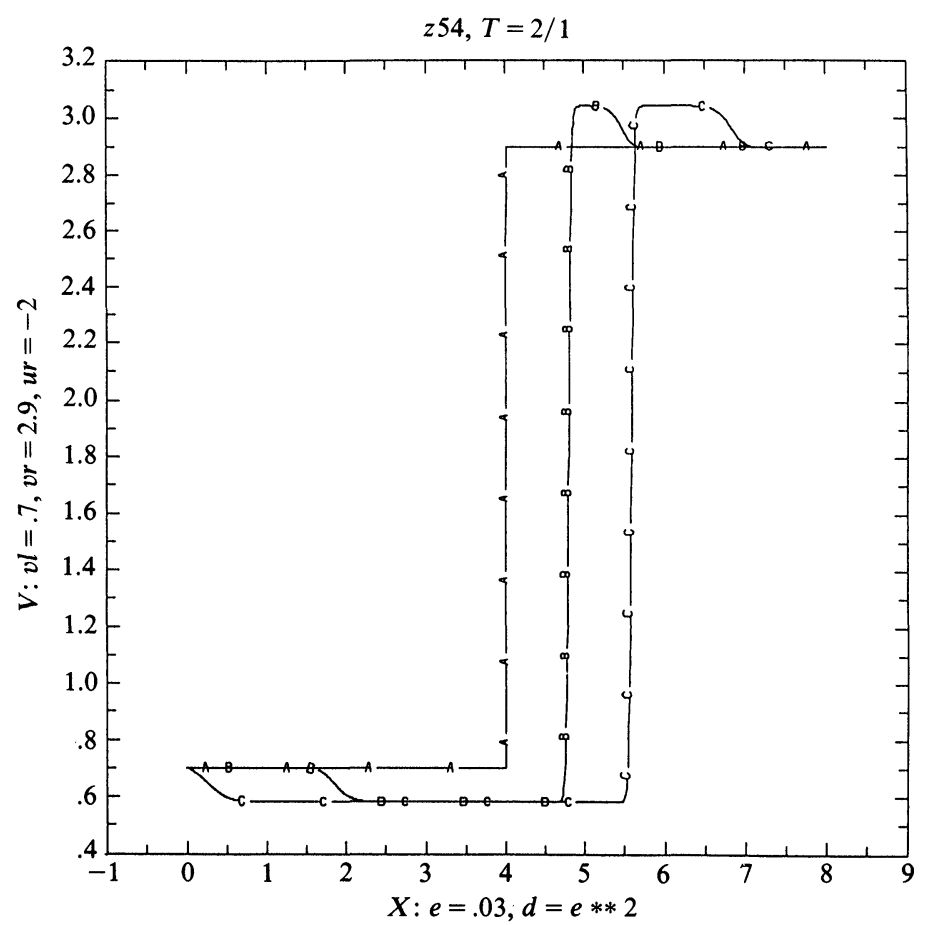

FIG. 12. A connection $S_{-}^{\varepsilon, \delta} S_{+j}^{\varepsilon, \delta} S_{+}^{\varepsilon, \delta}$. 


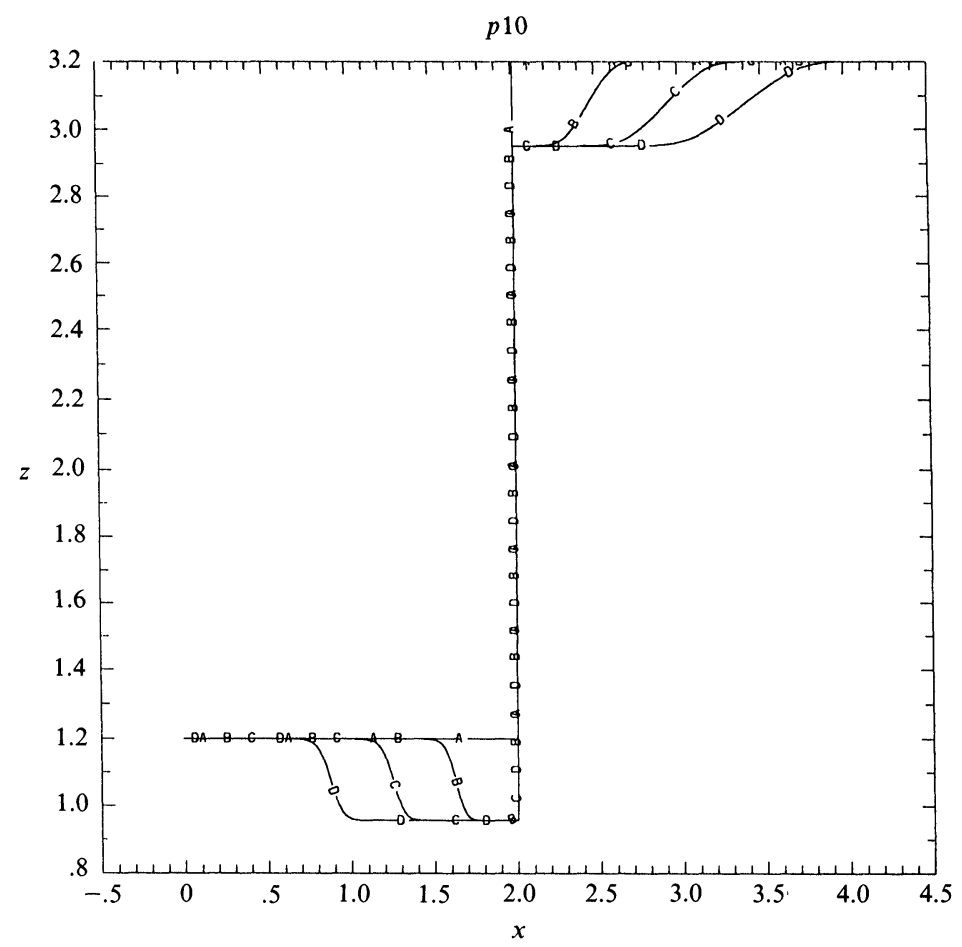

FIG. 13. A connection $S_{-}^{\varepsilon} S_{0}^{\varepsilon} R_{+}^{\varepsilon}$.

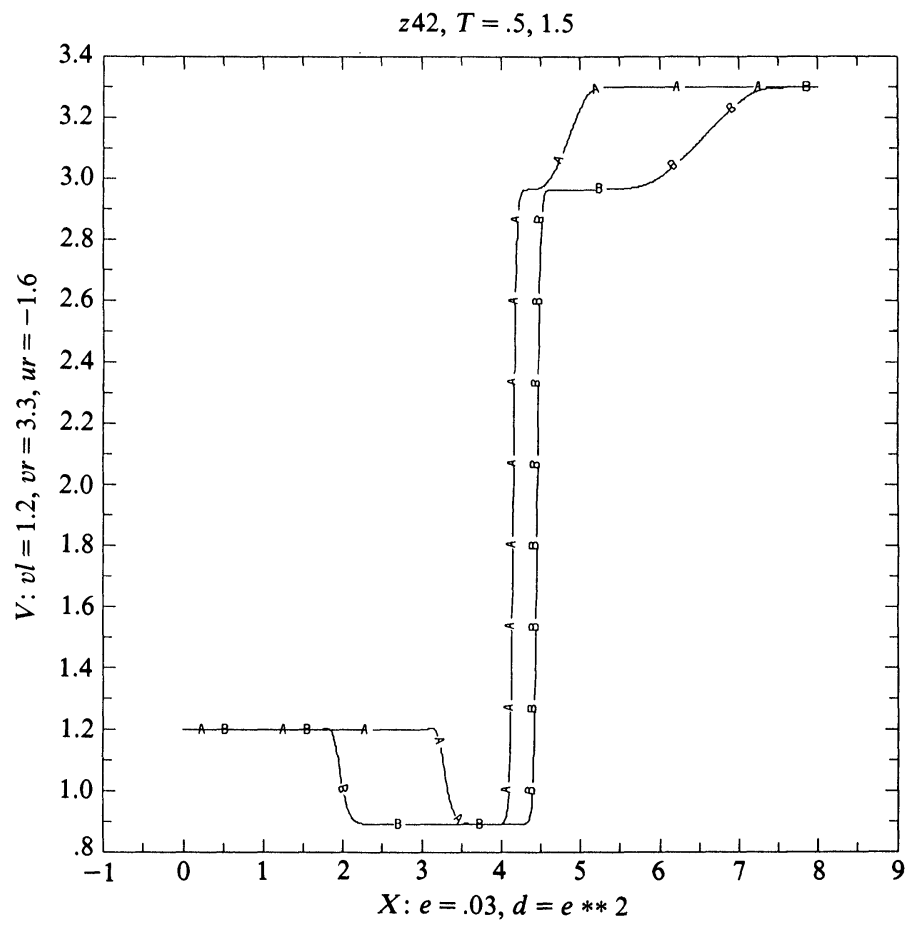

FIG. 14. A connection $S_{-}^{\varepsilon, \delta} S_{+j}^{\varepsilon, \delta} R_{+}^{\varepsilon, \delta}$. 
$\delta$ and an increasing function of $\delta$ for fixed $\varepsilon$. Moreover, for any choice of $\varepsilon, \delta$ the values of $U^{*}$ fill the geometric locus $W\left(U_{l}\right)$. So, if $\delta=0$ then $U^{*}=U_{s t}$ and if $\varepsilon=0$ then $U^{*}=U_{e q}$.

(3b) If $U_{l} \in D_{1}$, then $U^{*}$ is a decreasing function of $U_{l}$. However, $v^{*}$ is again decreasing with respect to $\varepsilon$ and increasing with respect to $\delta$ for fixed $U_{l}$, with the following property: If $\delta \neq 0$, then $U^{*}$ will fill the geometric locus $W\left(U_{l}\right)$. If $\delta=0$, any point on the Hugoniot curve with $\beta<v^{*}<v_{t}$ is allowed. If $\varepsilon=0$, then $U^{*}=U_{e q}$.

(4) If $\delta \neq 0$, then the only possible stationary phase jump is the connection $\langle m \rightarrow M\rangle$, i.e., the Maxwell equal area connection.

(5) If $\delta=0$, then any stationary phase jump is admissible, i.e., any connection of the type $\left\langle U_{l} \rightarrow U_{s t}\right\rangle$ is admissible. This case was treated by Pego [13] and Shearer [16]. The resulting solution satisfies the chord condition of Wendroff [25].

Now fix $U_{l}, \varepsilon$, and $\delta$. Then from part (3) there exists a unique state $U^{*} \in W\left(U_{l}\right)$ such that the connection $\left\langle U_{l} \rightarrow U^{*}\right\rangle$ is a forward phase jump. Choose $U_{r}$ to be on the curve $S^{+} R^{+}$passing through $U^{*}$; if $U_{r}$ is on $S^{+}$then $\left\langle U^{*} \rightarrow U_{r}\right\rangle$ is a forward shock. Now suppose $U_{l}$ and $U_{r}$ are the initial data, then the solution must be $S_{j}^{\varepsilon, \delta} S_{+}^{\varepsilon, \delta}$. To motivate this, assume that there is another state, say $U_{l 1} \in H_{l}$, such that $\left\langle U_{l 1} \rightarrow U_{r}\right\rangle$ is a phase jump. However, because of the monotonicity property ( $3 \mathrm{a}), v_{l 1}<v_{l}$ and $u_{l 1}>u_{l}$. Because the curve $S^{-} R^{-}$has a positive slope (see Fig. 2(a)), the state $U_{l 1}$ cannot be on the curve $S^{-} R^{-}$passing through the point $U_{l}$. Thus, $U_{l}$ cannot be connected to $U_{l 1}$ by $S_{-}^{\varepsilon, \delta}$ or $R_{-}^{\varepsilon, \delta}$ which is not consistent with part (2). This implies that $U_{r}$ cannot be connected directly to the other phase, so that there must be a state on $S^{+} R^{+}$through $U_{r}$ which will be connected to a point on the curve $S^{-} R^{-}$through $U_{r}$. But by (3a) and $(3 \mathrm{~b})$ such points must be $U_{l}$ and $U^{*}$. Similar reasoning for any of the initial data prescribed in this section argues that the solution is one of the 12 types listed in the classification.

(6) Finally, suppose $U_{l} \in D_{3}$. Then there is no admissible phase jump for $\delta \neq 0$ as shown by Slemrod [20], and the only possible connection $\left\langle U_{l} \rightarrow U_{r}\right\rangle$ for any $U_{r} \in H_{r}$ is $S_{-}^{\varepsilon, \delta} S_{j}^{\varepsilon, \delta} R_{+}^{\varepsilon, \delta}$. For $\delta=0$ the connection is either $S_{0}^{\varepsilon}$ or $S_{-}^{\varepsilon} S_{0}^{\varepsilon} R_{+}^{\varepsilon}$.

6. Stability of traveling wave solutions. In this section we investigate the asymptotic behavior (stability or instability) of traveling wave solutions for (1.1), as classified in $\S 5$, with perturbations in their initial data. Consider the initial data

$$
U(x, 0)=U_{0}(x)+V_{0}(x),
$$

in which the function $U_{0}(x)$ is a traveling wave solution of (1.1), and the function $V_{0}(x)$ is the initial perturbation, which is a function of small size decaying to zero at infinity.

Goodman [4], Kawashima and Matsumura [9], and Liu [11] have proved that shock profiles are stable with respect to small initial perturbations satisfying

$$
\int_{-\infty}^{+\infty}\left|\partial_{x}^{\alpha} W_{0}(x)\right|^{2} d x \leqq c, \quad V=\partial_{x} W_{0} ; \quad \alpha=0,1,2,
$$

provided that the system of equations is strictly hyperbolic and genuinely nonlinear with positive viscosity matrix on the right-hand side, and that the shocks are weak enough, i.e., $\left|v_{l}-v_{r}\right|$ is small enough. This result does not apply to the system (1.1), since it is of mixed type, the right-hand side includes a dispersion term in addition to the viscosity, and the phase jumps cannot be made weak. Our investigation of stability and instability is entirely numerical. 
6.1. Stability of constant states. Suppose that the initial data is

$$
U(x, 0)=\bar{U}+V_{0}(x)
$$

where $\bar{U}$ is a constant state lying in the hyperbolic region, and $V_{0}(x)$ satisfies (6.2). Following the computational results, several cases can be distinguished:

(1) If the initial perturbation (6.3) does not enter the elliptic region, i.e., $U(x, 0)$ is in the same phase as $\bar{U}$, then the solution of (1.1) with this initial data consists of two trains of $N$-waves moving in opposite directions along the characteristics of (1.1). The $N$-wave structure is due to the nonlinearity of the pressure $p(v)$. If $\delta=0$, it is known that these waves will decay to the constant state $\bar{U}$ with rate $O\left(t^{-1 / 2}\right)$ because of the viscosity term. For $\delta \neq 0$, our numerical computations also show decay of the $N$-waves. Therefore, in this case the constant state $\bar{U}$ is stable.

(2) If the initial perturbation enters the elliptic region, then the solution $U(x, t)$ will cross into the other phase at least for a short time. Such large perturbations will be called elliptic perturbations. As time progresses, the solution of this system may behave in two different ways.

First, the solution may pull back to the base state $\bar{U}$ splitting into two $N$-waves which decay to zero. In this case $\bar{U}$ is stable. Second, after a long time the solution may asymptotically approach a new structure connecting the two different phases. In this case $\bar{U}$ is unstable. This stability (or instability) of the solution depends on the value of the specific volume $v$, the viscosity $\varepsilon$, the dispersion coefficient $\delta$, and the form of the perturbation in the elliptic region.

These two types of behavior occur in different regions of phase space, as described next:

(2a) If the base state $\bar{U}$ is in the region $D_{1}$, i.e.,

$$
v<\gamma \text { or } v>\sigma
$$

then the solution of the system (1.1) is always stable. Therefore, we call $D_{1}$ the globally attractive region.

(2b) If the base state $\bar{U}$ is in $D_{2}$, i.e.,

$$
\gamma \leqq v \leqq m \text { or } \quad M \leqq v \leqq \sigma,
$$

then the solution of the system (1.1) is stable to any initial perturbations, if this system is dispersive $(\delta \neq 0)$. The asymptotic stability means that the solution converges to the constant state, i.e., $\lim _{t \rightarrow \infty} U(x, t)=\bar{U}$, as in Fig. 15. If this system has no dispersion $(\delta=0)$ then the solution is unstable, and does not converge to a constant state. However, even in this case, it does not grow indefinitely. Instead, the solution converges to a combination of stationary phase jumps connecting $\bar{U}$ to the other phase, as seen in Fig. 16. Therefore, we call $\mathrm{D}_{2}$ the dispersively stable region.

(2c) If the base state $\bar{U}$ is in the metastable region, i.e.,

$$
m<v<\alpha \text { or } \beta<v<M,
$$

stability depends on the form of the perturbation in the elliptic region.

(2ci) The base state $\bar{U}$ is unstable when the perturbation has more crests than troughs in the elliptic region. This perturbation will be called an effective-elliptic perturbation. If $\delta \neq 0$, then

$$
\lim _{t \rightarrow \infty} U(x, t)=S_{-}^{\varepsilon, \delta} S_{-j}^{\varepsilon, \delta} S_{+j}^{\varepsilon, \delta} S_{+}^{\varepsilon, \delta},
$$

as illustrated in Fig. 17. If $\delta=0$, then

$$
\lim _{t \rightarrow \infty} U(x, t)=S_{0} S_{0} \cdots S_{0} .
$$



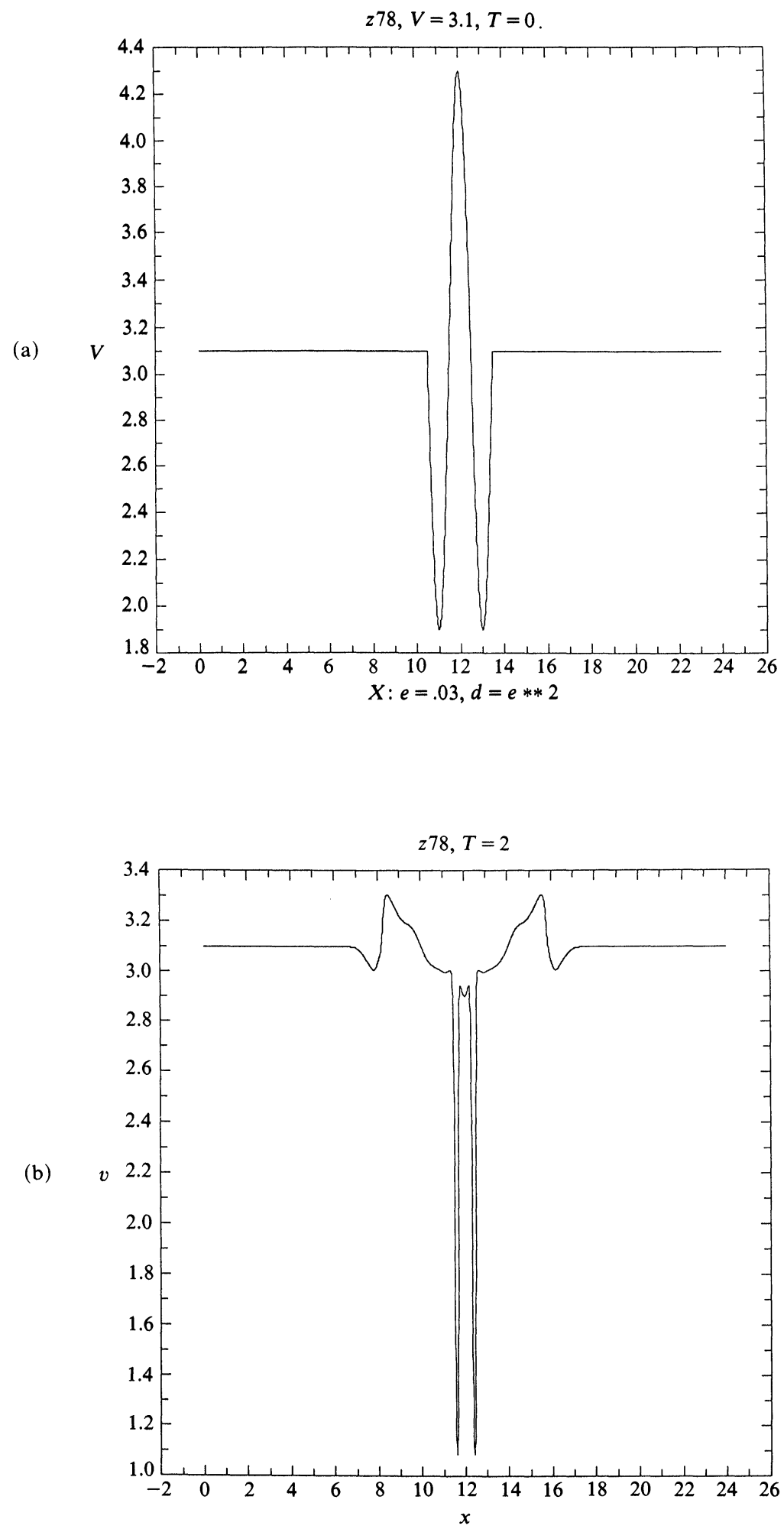

FIG. 15. A constant state $\bar{U}$ in $D_{2}$ for $\delta \neq 0$ with an elliptic perturbation. At three different times (a) $T=0$, (b) $T=2$, (c) $T=5$. 


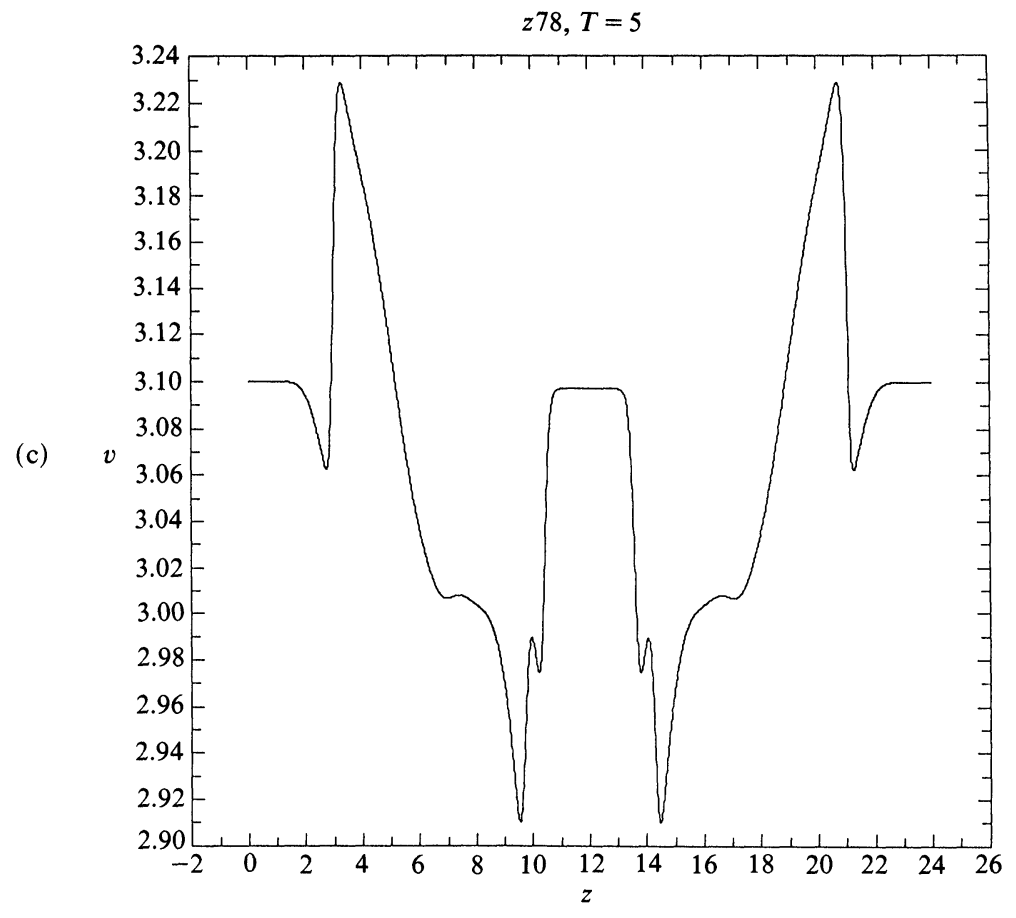

FIG. 15 (continued).

(2cii) The solution is stable if there are more troughs than crests in the elliptic region.

Note that in all the cases above, the structure of the unstable solution in the case of the viscous system $(\delta=0)$ is $\left\langle S_{0} S_{0} \cdots S_{0}\right\rangle$, and in the case of the dispersive system $(\delta \neq 0)$ the structure of the unstable solution is $\left\langle S_{-} S_{-j} S_{+j} S_{+}\right\rangle$.

Next, consider the stability of nonconstant traveling wave solutions. We now distinguish three types of traveling wave solutions.

6.2. Stability of monotone shock profiles. Viscous shock profiles are monotone solutions connecting two end states which are in the same phase. Such solutions are stable with respect to infinitesimal perturbations. The perturbations result in two decaying diffusive $\mathrm{N}$-waves, in agreement with Liu's analysis of shock wave stability. However, if the perturbations are effective-elliptic perturbations, then the shock profiles may be unstable and approach another combination of shock profiles. Such instability will occur if the following conditions are satisfied:

(1) If the system $(1.1)$ is dispersive $(\delta \neq 0)$, and the two end states $U_{l}$ and $U_{r}$ are in the metastable region $D_{3}$, then the shock profiles $S_{-}^{\varepsilon, \delta}$ or $S_{+}^{\varepsilon, \delta}$ are unstable for effective-elliptic perturbations. The solution of (1.1) connecting the two end states will approach a connection of the type $S_{-}^{\varepsilon, \delta} S_{-j}^{\varepsilon, \delta} S_{+j}^{\varepsilon, \delta} S_{+}^{\varepsilon, \delta}$ as shown in Fig. 18.

(2) If the system (1.1) is viscous $(\delta=0)$ and the two end states are in $D_{2} \cup D_{3}$, then the shock profiles are unstable for effective-elliptic perturbations. The asymptotic solution is of the following type $S_{-}^{\varepsilon} S_{0} S_{0} \cdots S_{0}$, or $S_{0} S_{0} \cdots S_{0} S_{+}^{\varepsilon}$ as shown in Fig. 19.

All other monotone shock profiles are globally stable with respect to any type of perturbation.

6.3. Stability of oscillatory shock profiles. Oscillatory shock profiles connecting two end states in the same phase, which occur for large values of $\delta$, are stable with 

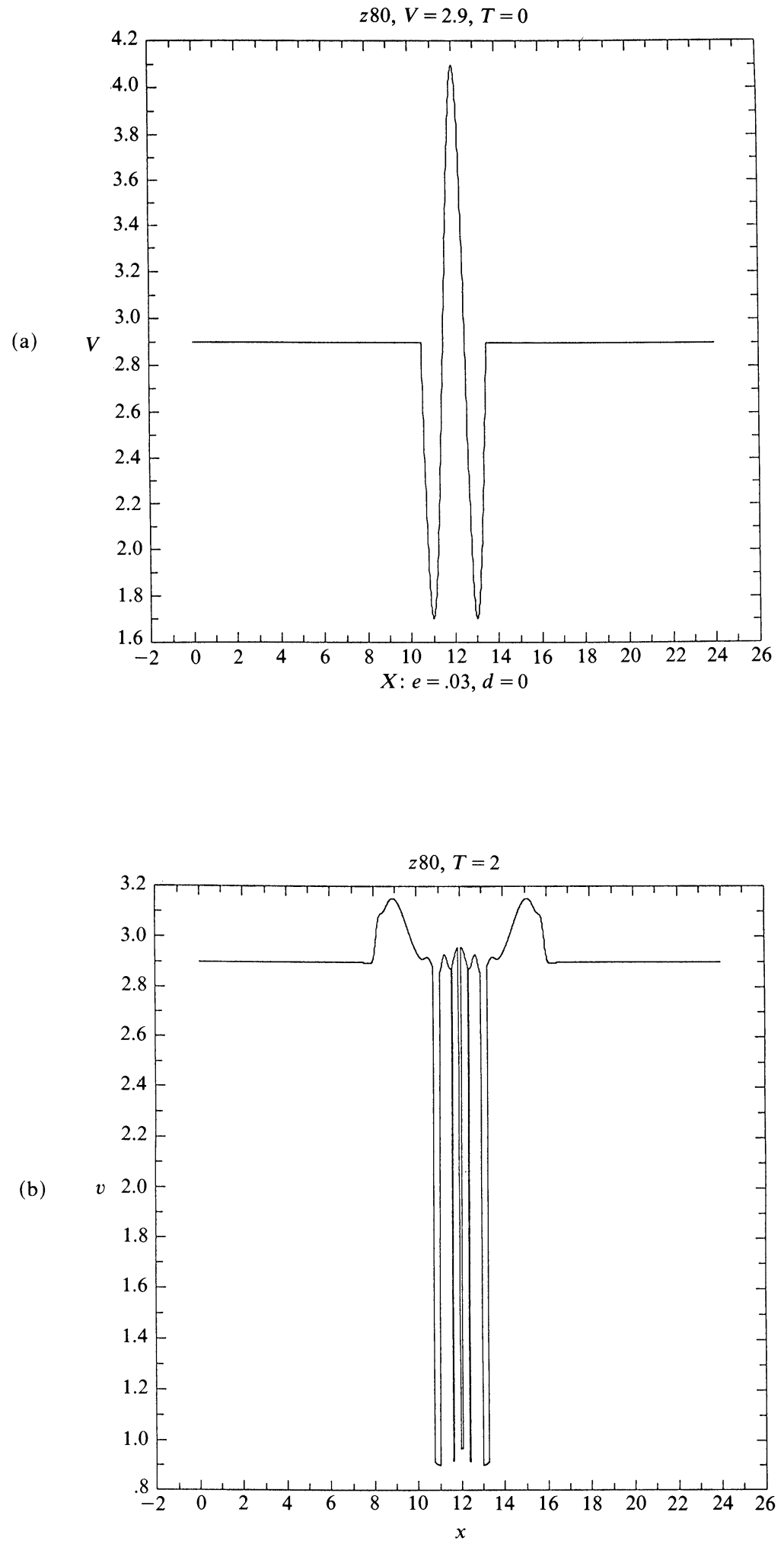

FIG. 16. Same as Fig. 15 for $\delta=0$. 


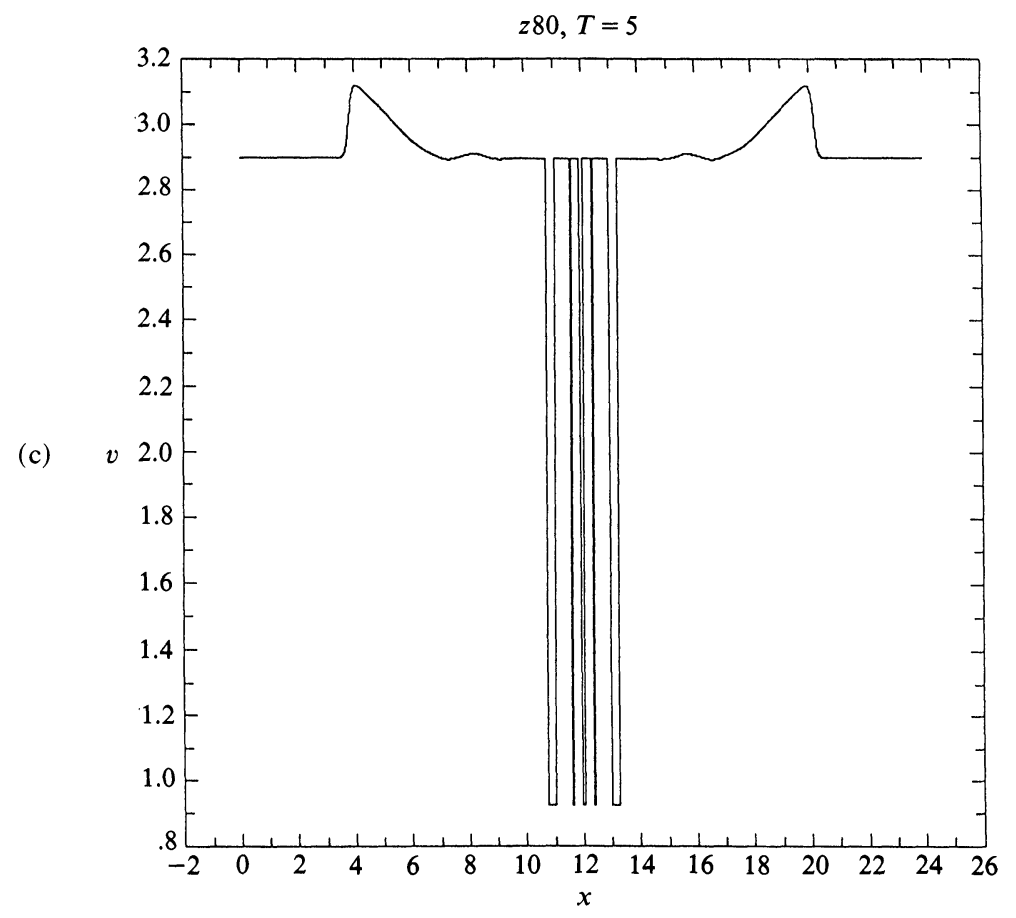

FIG. 16 (continued).

respect to any perturbation that does not enter the elliptic region. If the oscillatory solution connects to a state that is very close to the elliptic region, then the oscillations will enter the elliptic region. Then one would expect that this solution would become unstable. However, this oscillatory solution is found to be stable with respect to infinitesimal perturbations, despite their entering into the elliptic region. The oscillatory shock profile behaves like the viscous shock profile for effective-elliptic perturbations.

6.4. Stability of phase jumps. Phase jumps are solutions $S_{j}^{\varepsilon, \delta}$ connecting two states located in different phases and crossing the unstable (elliptic) region. Numerical computations show that solutions are stable for any initial perturbations and that the perturbations only result in the formation of two decaying $N$-waves.

Figure 20 shows a phase jump connecting a state in the metastable region, and we might expect that adding an effective-elliptic perturbation would lead to instability. This is seen for a short time, but as time increases the short time instability disappears as a result of the interaction with the moving phase jump, and two decaying $N$-waves form. This shows that phase jumps are stable solutions of system (1.1).

6.5. Width of phase jumps. The stability of the phase jump can be understood by comparing its width to the unstable wavelengths for (1.1). If the unstable wavelengths were much smaller than the phase jump width $w$, then the phase jump would be unstable. However, as shown below, the $w$ is much smaller than the unstable wavelengths, so that the phase jump can be stable.

Suppose the function $u(x)$ is the phase jump. Its width $w$ may be defined as

$$
w=\frac{\left|u_{l}-u_{r}\right|}{\max \left|u^{\prime}(x)\right|}
$$



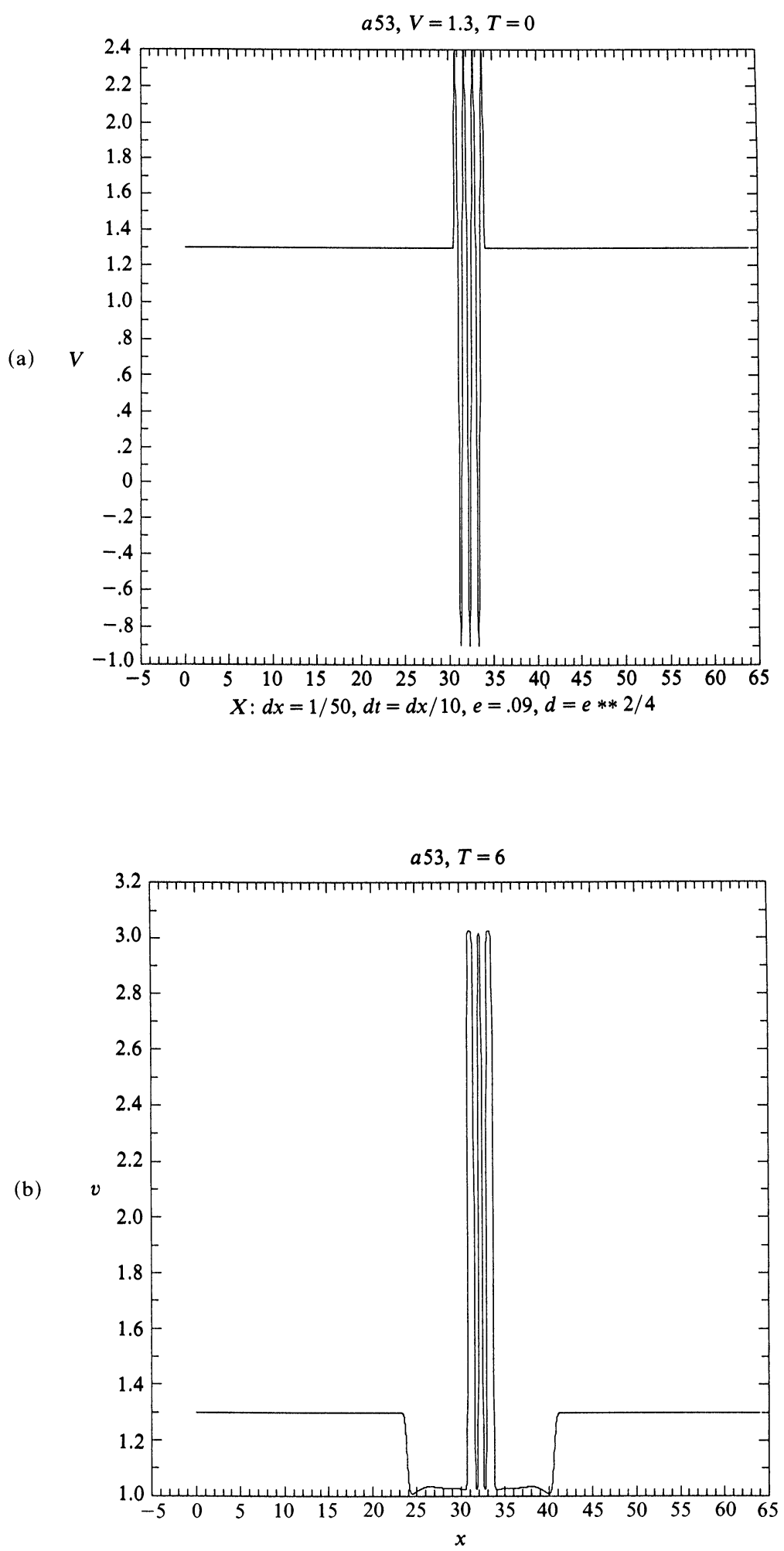

FIG. 17. A constant $\bar{U}$ in the metastable region for $\delta \neq 0$ with a strong elliptic perturbation at times (a) $T=0$, (b) $T=6$, (c) $T=15,30$. 


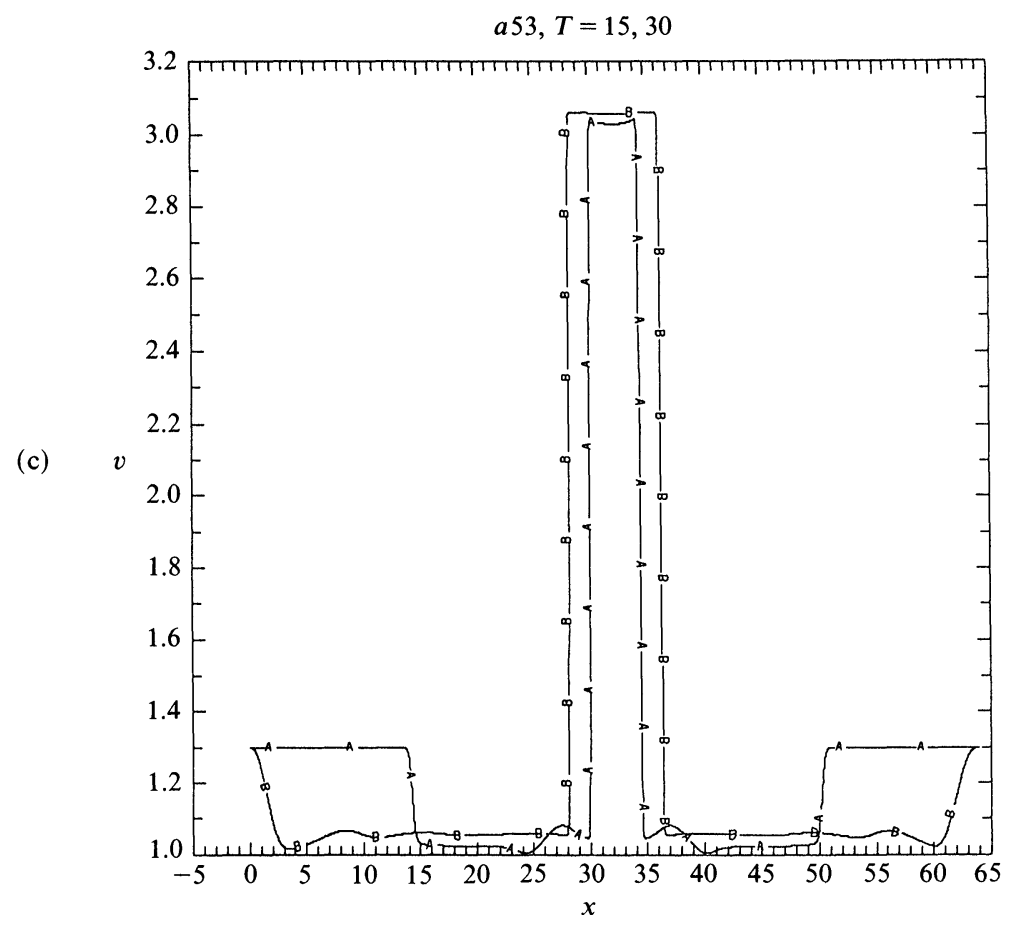

FIG. 17 (continued).

If $k_{s}$ is a typical wave number for the function $u^{\prime}(x)$, then

$$
w=O\left(1 / k_{s}\right) \text {. }
$$

The linearized analysis of $\S 2$ is valid for wave numbers $k$ for which the phase jump looks constant, i.e., for $|k| \gg\left|k_{s}\right|$. We know from $\S 2$ that the interval of unstable wave numbers is $|k|<k_{0}=\sqrt{p^{\prime}} \delta^{-1 / 2}$. Thus, linear analysis shows instability of the phase jump if there are wave numbers $k$ satisfying both of these criteria, which is possible if $k_{0} \gg k_{s}$, i.e., if

$$
w \gg \delta^{1 / 2} .
$$

Based on numerical results, we establish the following approximate formula for the width of the phase jumps $S_{j}^{\varepsilon, \delta}$ :

1. If $U_{l} \in D_{2} \cup D_{3}$, then

$$
w=c \sqrt{\delta},
$$

where $c$ is a positive constant. This confirms a remark by Pego [13] that interphase shocks approach zero thickness as the shock speed approaches zero. Therefore, $k_{m} \approx k_{s}$ and thus the phase jumps should be stable, as we have already established numerically.

2. If $U_{l} \in D_{1}$, then

$$
w=\frac{c(\varepsilon, s)}{s} \sqrt{\delta}
$$

where $s$ is the speed of the phase jump (which is not near 0 ) and the function $c(\varepsilon, s)$ is $o(s)$ as $s \rightarrow+\infty$. This again shows that the phase jump width tends to zero as the speed increases. Again the instability condition (6.6) cannot be satisfied for phase jumps. This gives a partial analytical justification for the stability of the phase jumps. 
$a 33, v l=1.4, v r=1.1, S_{-}, T=0$

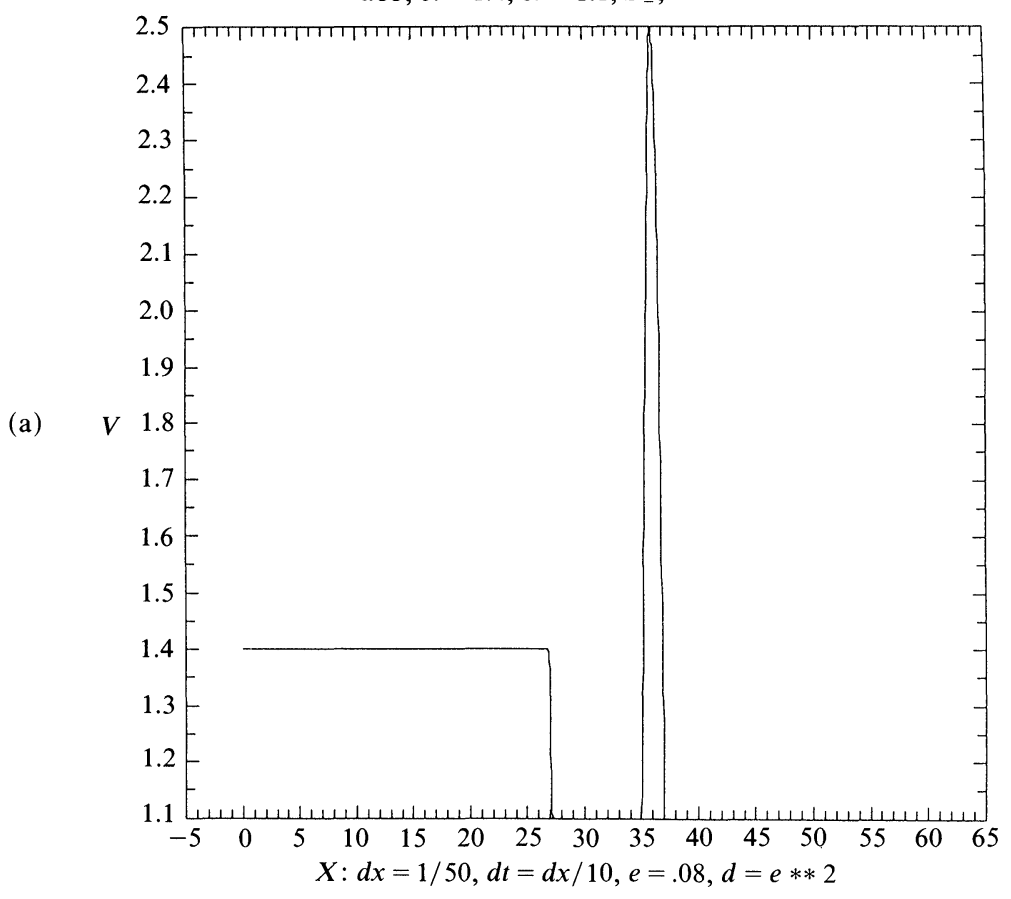

a33, $T=10,20$ i

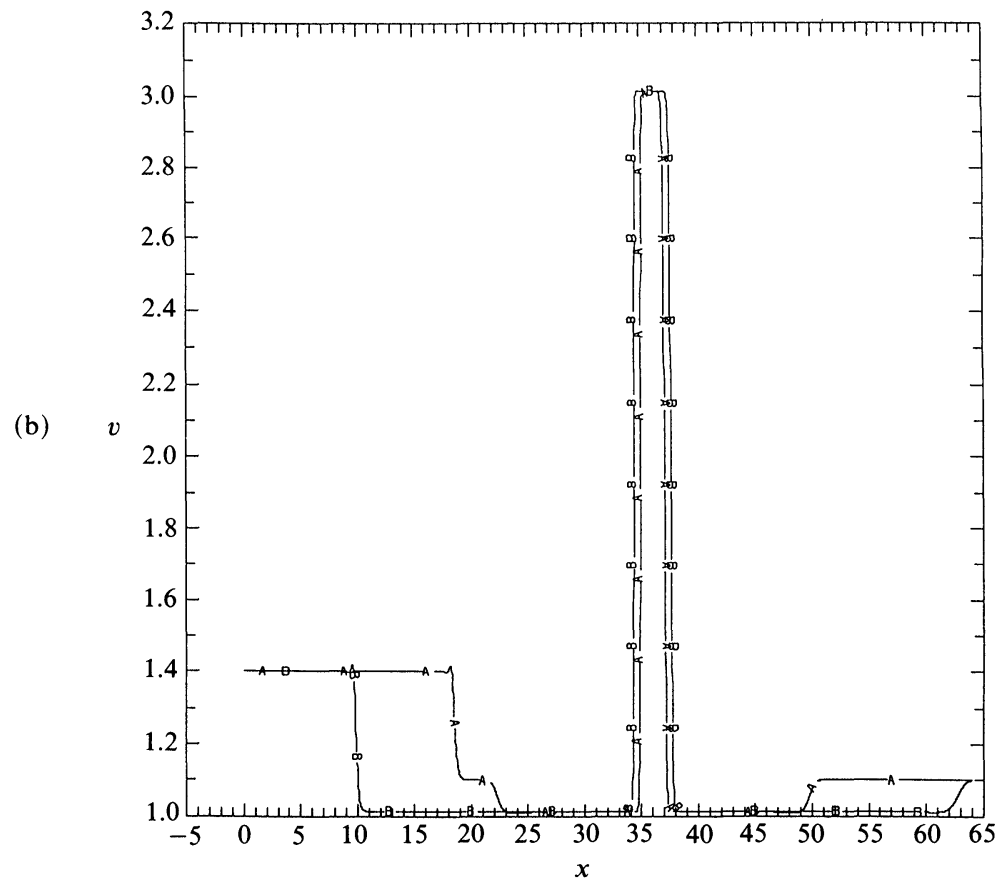

FIG. 18. A strong elliptic perturbation of a monotone shock wave for $\delta \neq 0$ at times (a) $T=0$, (b) $T=10,20$. 

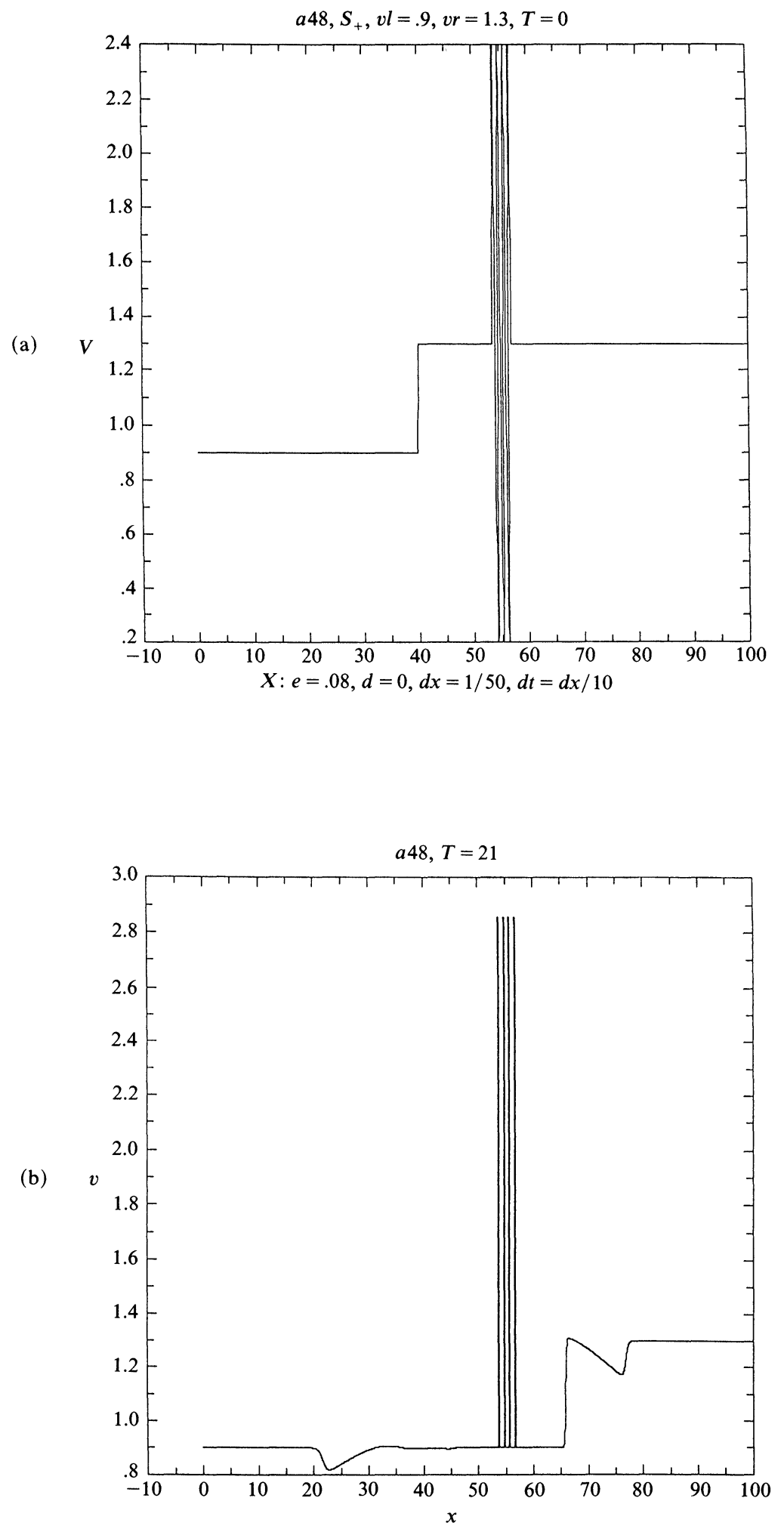

Fig. 19. Same as Fig. 18 with $\delta=0$ at times (a) $T=0$, (b) $T=21$. 

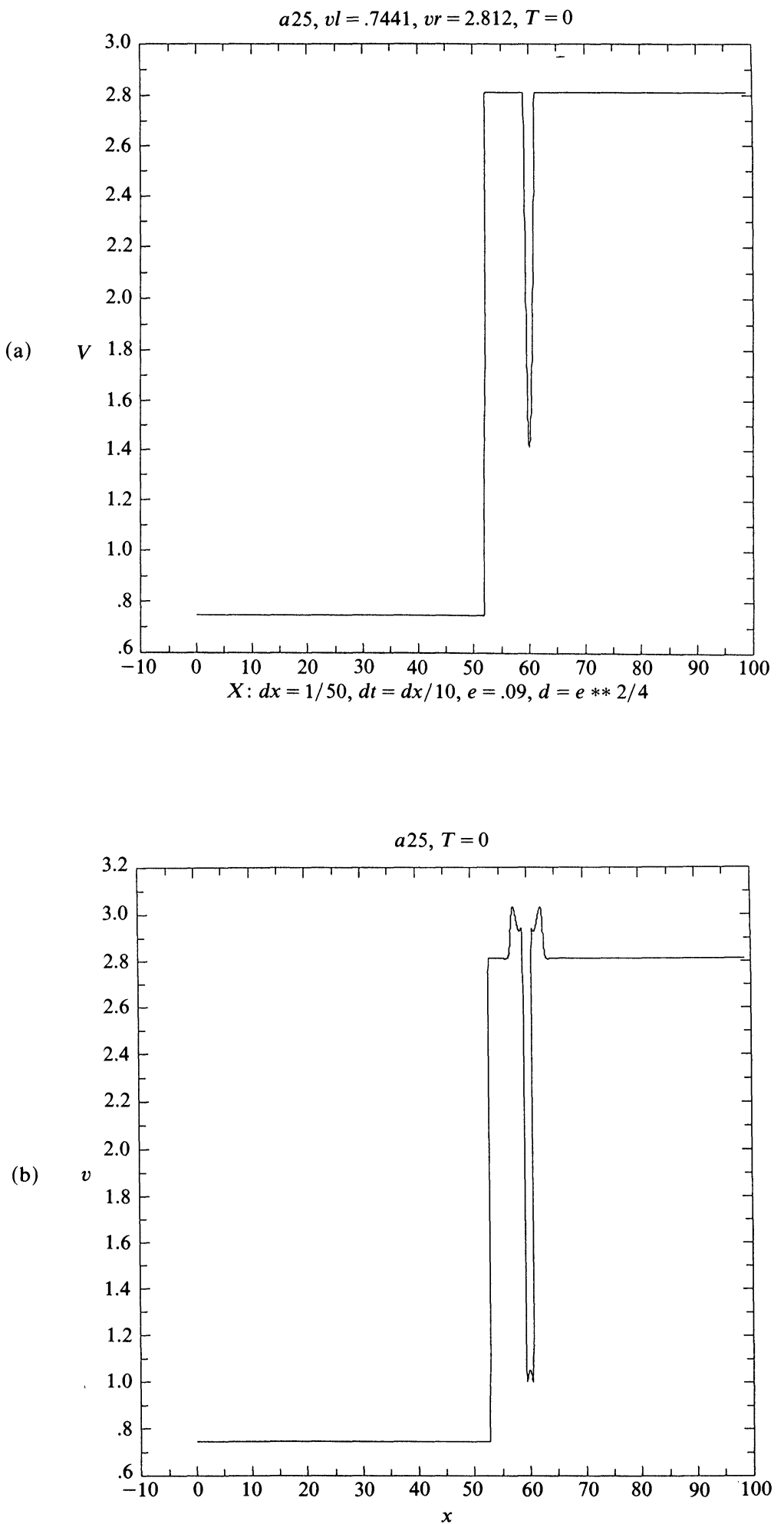

FIG. 20. A strong elliptic perturbation of a phase jump with end point in the metastable region at (a) $T=0$, (b) $T=2$, (c) $T=10$, (d) $T=24,30$. 

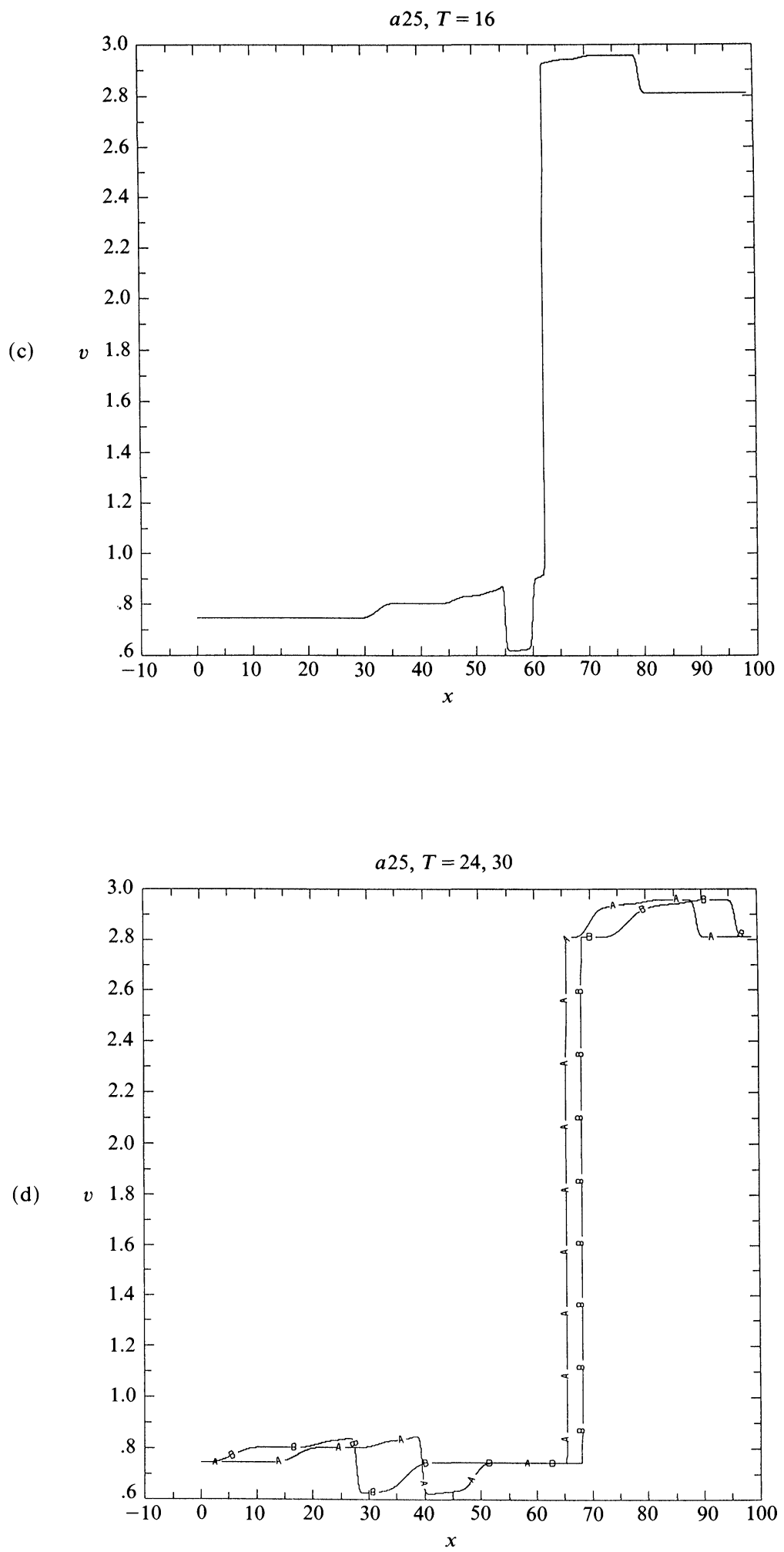

FIG. 20 (continued). 


\section{REFERENCES}

[1] V. Bongiorno, L. E. Scriven, And H. T. Davis, Molecular theory of fluid interfaces, J. Colloid Interface Sci., 57 (1976), pp. 462-475.

[2] B. U. Felderhof, The gas-liquid interface near a critical point, Phys., 48 (1970), pp. 541-560.

[3] J. Glimm, The continuous structure of discontinuities, preprint.

[4] J. GoOdman, Nonlinear asymptotic stability of viscous shock profiles for conservation laws, Arch. Rational Mech. Anal., 95 (1986), pp. 325-344.

[5] R. Hagan AND M. Slemrod, The viscosity-capillarity criterion for shocks and phase transitions, Arch. Rational Mech. Anal., 83 (1983), pp. 333-361.

[6] H. HATTORI, The Riemann problem for a van der Waals fluid with entropy rate admissibility criterionisothermal case, Arch. Rational Mech. Anal., 92 (1986), pp. 247-263.

[7] - The Riemann problem for a van der Waals fluid with entropy rate admissibility criterionnonisothermal case, J. Differential Equations, 65 (1986), pp. 158-174.

[8] A. V. KAZHik hov AND V. B. NikolaEV, On the correctness of boundary value problems for the equations of a viscous gas with nonmonotone state function, Amer. Math. Soc. Transl., 125 (1985), pp. 45-50.

[9] S. Kawashima And A. Matsumura, Asymptotic stability of traveling wave solutions of system for one-dimensional gas motion, Comm. Math. Phys., 101 (1985), pp. 97-127.

[10] D. J. KorteweG, Sur la forme que prennent les équations des mouvements des fluides si l'on tient compte des forces capillaires par des variations de densité, Arch. Neerl. Sci. Exactes Nat. Ser. II, 6 (1901), pp. 1-24.

[11] T. P. LIU, Nonlinear stability of shock waves for viscous conservation laws, Mem. Amer. Math. Soc., 328 (1985), pp. 1-108.

[12] B. Nicolaenko, private communication.

[13] R. PEGO, Phase transitions in one-dimensional nonlinear viscoelasticity: admissibility and stability, Arch. Rational Mech. Anal., 97 (1987), pp. 353-394.

[14] D. G. SCHAEFFER AND M. SHEARER, The classification of $2 \times 2$ systems of non-strictly hyperbolic conservation laws, with application to oil recovery, Comm. Pure Appl. Math., 40 (1987), pp. 141-178.

[15] M. SHEARER, The Riemann problem for a class of conservation laws of mixed type, J. Differential Equations, 46 (1982), pp. 426-443.

[16] - Admissibility criteria for shock wave solutions of a system of conservation laws of mixed type, Proc. Roy. Soc. Edinburgh, 93A (1983), pp. 233-244.

[17] - Nonuniqueness of admissible solutions of Riemann initial value problems for a system of conservation laws of mixed type, Arch. Rational Mech. Anal., 93 (1986), pp. 45-59.

[18] M. Shearer, D. G. Schaeffer, D. Marchesin, And P. L. Paes-Leme, Solution of the Riemann problem for a prototype $2 \times 2$ system of non-strictly hyperbolic conservation laws, Arch. Rational Mech. Anal., 97 (1987), pp. 299-320.

[19] M. Slemrod, Admissibility criteria for propagating phase boundaries in a van der Waals fluid, Arch. Rational Mech. Anal., 81 (1983), pp. 301-315.

[20] - Dynamic phase transitions in a van der Waals fluid, J. Differential Equations, 52 (1984), pp. 1-23.

[21] M. Slemrod and J. E. Flaherty, Numerical integration of a Riemann problem for a van der Waals fluid, preprint.

[22] M. SlemRod And J. MARSDEn, Temporal and spatial chaos in a van der Waals liquid due to periodic thermal perturbations, Adv. Appl. Math., 6 (1985), pp. 135-158.

[23] J. SMOller, Shock Waves and Reaction-Diffusion Equations, Springer-Verlag, New York, Berlin, 1983.

[24] V. A. Solonnikov AND A. V. Kazhikhov, Existence theorems for the equations of motion of a compressible viscous fluid, Ann. Rev. Fluid Mech., 13 (1981), pp. 79-95.

[25] B. WendrofF, The Riemann problem for materials with nonconvex equation of state, J. Math. Anal. Appl., 38 (1972), pp. 454-466.

[26] G. B. Whitham, Linear and Nonlinear Waves, John Wiley, New York, 1974. 